\title{
Understanding Extended Projected Profile (EPP) Trajectory Error Using a Medium-Fidelity Aircraft Simulation
}

\author{
Nelson M. Guerreiro ${ }^{1}$ and Matthew C. Underwood ${ }^{1}$ \\ NASA Langley Research Center, Hampton, VA 23681, USA
}

\begin{abstract}
A critical component of Trajectory-Based Operations is the ability for a consistent and accurate 4-dimensional trajectory to be shared and synchronized between airborne and ground systems as well as amongst various ground automation systems. The Aeronautical Telecommunication Network-Baseline 2 standard defines the Extended Projected Profile (EPP) trajectory that can be sent via Automatic Dependent Surveillance-Contract from an aircraft to ground automation. The EPP trajectory message contains a representation of the reference trajectory from an aircraft's Flight Management System (FMS). In this work, a set of scenarios were run in a medium-fidelity aircraft and FMS simulation to perform an initial characterization of EPP trajectory errors under a given set of conditions. The parameters investigated were the route length, route type, wind magnitude error, wind direction error, and with and without a required time-of-arrival constraint.
\end{abstract}

\section{Introduction}

$\mathrm{T}$ Trajectory-based Operations (TBO) are a key component of the Next Generation Air Transportation System (NextGen) [1, 2]. TBO are based on the premise that, in the near future, aircraft operating in the National Airspace System (NAS) will be managed more strategically based on a four-dimensional trajectory (4DT). A 4DT defines the lateral, vertical, and temporal path of an aircraft and can be computed by automation, computed by the aircraft, or synchronized between these two agents. TBO extends this premise by providing separation, sequencing, spacing, and merging services to flights based on these 4DTs, thus providing efficiency, capacity, and safety benefits [2].

A critical component of TBO is the ability for a consistent and accurate 4DT representation to be shared and synchronized between airborne and ground systems as well as amongst various ground automation systems. Sharing of the 4DT is important to ensure that all stakeholders of a given flight have a common baseline from which to build situation awareness of the present and future states of any aircraft at any time during the flight. Additionally, through management and sharing of the 4DT, preferences regarding how the trajectory of a given flight should be modified can be developed and shared with other stakeholders of the flight. Furthermore, for the Air Navigation Service Provider (ANSP), sharing the 4DT amongst various ground-based automation platforms assists in system-wide benefit realization by considering the widespread system-level impacts that result from specific decisions.

In today's NAS, no comprehensive trajectory information is broadcast from the flight deck to ground-based automation systems. Several ground-based automation platforms have independent, domain-specific trajectory generators that build trajectory representations based on constraints in the NAS. These systems typically do not share their trajectory representations, and if they do, the information that is passed to other systems is usually inadequate (constraints with insufficient detail) and is only passed in one direction with no opportunity for negotiation. This leads to un-coordinated decision making without the input of all stakeholders.

The Aeronautical Telecommunication Network-Baseline 2 (ATN-B2) standard [3] defines the Extended Projected Profile (EPP) trajectory that can be sent via Automatic Dependent Surveillance-Contract (ADS-C) from an aircraft to ground automation. The EPP trajectory message contains a representation of the reference trajectory from an aircraft's Flight Management System (FMS). The EPP is included in the ATN-B2 standard as one method for

\footnotetext{
${ }^{1}$ Research Aerospace Engineer, Crew Systems and Aviation Operations Branch, MS 152, AIAA Member.
} 
facilitating trajectory synchronization between airborne and ground systems. As of 2017, the ATN-B2 standard has been mandated by EUROCONTROL [4], but not by the Federal Aviation Administration (FAA).

Several studies $[5,6,7,8,9]$ have demonstrated potential uses for, and illustrated shortcomings of, the data contained within the EPP trajectory. However, none of these studies has attempted to characterize the EPP error that results from an aircraft's own trajectory prediction error. The objective of this analysis and paper is to produce an initial characterization of the accuracy of the EPP message under a limited set of conditions. This is intended to be an initial characterization that produces an order of magnitude understanding about the potential limitations in the use of the shared EPP trajectory information.

\section{Background}

\section{A. Overview of ADS-C and EPP}

ADS-C is an application that provides automatic reports from an aircraft's FMS to an ANSP. ADS-C reports can be requested by the ANSP in one of three types of contracts - an on-demand one-time report, a periodic contract, and an event-driven contract - and the aircraft system is capable of providing ADS-C reports to meet these contract requests. The ADS-C report's content, and the conditions under which the report is sent, vary depending on the type of contract request and the conditions specified in the request [3]. Only one contract of a given type can be set up per aircraft with any given ANSP.

The EPP trajectory is one of the many data elements that can be included in an ADS-C report. It provides a set of trajectory points that are a representation of the aircraft's four-dimensional FMS reference trajectory. It extends and improves upon the Intermediate Projected Intent (IPI) trajectory downlink message, which was available in Future Air Navigation Systems (FANS) 1/A. The EPP message contains information based on the route and performance data that has been entered into the aircraft's Flight Management System (FMS), including up to 128 trajectory change points (TCPs) and constraints (e.g., altitude, time, etc.) at each waypoint $[3,7]$. Figure 1 defines the data contained in the EPP message. Note that the minimum required set of data for an EPP trajectory point is the latitude and longitude position.

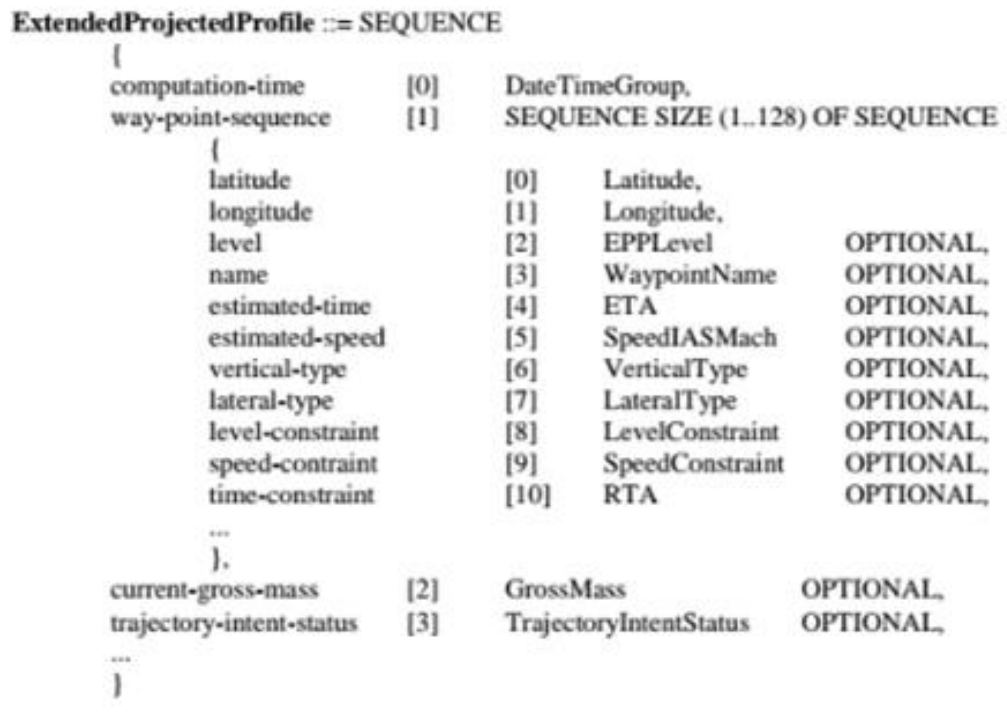

Figure 1. ADS-C EPP message contents [3].

\section{B. Use Cases for EPP}

Data contained within the EPP message are anticipated to facilitate trajectory synchronization between aircraft and ANSP automation, as well as to provide data required for automated conformance monitoring. As stated by Bronsvoort, et al in [10], "The goal of air-ground trajectory synchronization is to produce trajectories in disparate systems whose discrepancies are operationally insignificant, increasing the likelihood of flying the planned conflictfree and preferred trajectories." Trajectory synchronization is anticipated to occur through several means, including augmentation of ground-based trajectory generation systems with FMS-calculated and inferred intent data from the EPP message, and calibration of the ground-based trajectory generators utilizing data contained within the EPP message. Once the trajectories are synchronized, they can be used for conformance monitoring.

A common requirement among many Decision Support Tools (DSTs) used in air traffic management is the ability to accurately, and timely, predict an aircraft's future state. However, if any of the trajectory prediction inputs have large uncertainty (e.g., weather forecasts), or are incorrect (e.g., tactical vectoring not entered into ground automation), the resulting trajectory predictions can be un-reliable. Mondoloni and Bayraktutar state that the intent error caused by vectoring is among the highest-impact factors that affect prediction accuracy, due to the significant effects on cross- 
track and along-track error [11]. Furthermore, regarding longitudinal uncertainty, ground-based trajectory generators often have inadequate knowledge of an aircraft's speed schedule and weight, which results in inaccuracies in the climb and descent portions of the flight $[6,12]$.

In order to address trajectory uncertainty, work conducted by Paglione, et al [13], and Bronsvoort, et al [14], demonstrated that ground-based trajectory generation systems can benefit significantly from using data from the aircraft's FMS-generated trajectory (e.g., via the EPP message). Bronsvoort, et al [10], describe two approaches for using such information. The first approach is to employ the EPP message directly as the trajectory to be used by ANSP DSTs; however, arguments have shown that this is not the most practical use for the EPP message. The second approach is to augment the data used for ground-based trajectory generation with data contained in the EPP message. Because the FMS is the most accurate source of aircraft intent and other parameters required for trajectory generation, it follows that trajectory predictors in ground systems can improve accuracy when using EPP-derived intent and parameters [10]. Haugg, et al [8], state that the most beneficial data taken directly from the EPP message for a groundbased trajectory generator is the speed profile, especially the climb Calibrated Airspeed (CAS) or Mach number. Šošovička, et al [5], re-iterate the findings of Haugg, et al, and posit that the highest benefit for the ground-based trajectory predictors occurs when the aircraft-derived data from the EPP message is used in conjunction with knowledge of how the aircraft is handled in any given sector (e.g., hand-off altitudes, entry/exit points).

In addition to its usefulness in deriving aircraft intent, the EPP can be used to calibrate aircraft performance characteristics unknown to the ground-based trajectory generator. This allows for synchronization of the current trajectory with the trajectory prediction process [10]. Bronsvoort, et al [9], demonstrated that the EPP message could be successfully used to determine an aircraft performance model calibration function that accounts for any variables not specifically recorded in the EPP. With trajectory predictor calibration, a ground-based trajectory predictor can perform high accuracy "what-if" computations that anticipate the FMS behavior upon changes in aircraft intent.

The EPP can also play a crucial role in conformance monitoring. An envisioned ANSP conformance monitoring DST uses surveillance reports and alerts the controller when the aircraft is outside the conformance margins allowed by the clearance.

\section{Limitations of EPP}

Although the EPP message is regarded in the literature as the practical solution for achieving trajectory synchronization, there are some shortcomings within the message standard. For example, the EPP message contains turn geometry information for fly-by turns and supports Radius-to-Fix (RF) legs. However, it does not contain turn geometry information for fly-over waypoints. Mondoloni and Bayraktutar include turn modeling in their list of highimpact factors that affect prediction accuracy [11]. Bronsvoort, et al [7], discuss the impacts of the lack of turn radii for a fly-over waypoint in the EPP message standard. Because the turn radii information is not in the EPP message for fly-over waypoints, assumptions need to be made when generating the lateral trajectory, which could result in significant lateral and longitudinal trajectory error. Additionally, while the EPP message includes data regarding the aircraft's current gross mass (i.e.; the mass of the aircraft at the time when the reference trajectory used for the EPP message was generated), it does not provide information about predicted fuel burn. Mondoloni and Bayraktutar also include lack of aircraft weight in their list of high-impact factors that affect prediction accuracy [11].

In order to reduce complexity and bandwidth, the EPP message does not include information about the temperature and wind forecast that the FMS used for trajectory calculations, nor does it include the actual temperature and winds that the aircraft is experiencing at the time the trajectory calculation is performed $[9,10]$. Mondoloni and Bayraktutar include lack of wind forecast and wind gradient modeling in their list of high-impact factors that affect prediction accuracy [11]. Complicating matters further, Bronsvoort, et al, highlight the differences between weather information accessible by ground-based trajectory generators and the FMS, as well as differences between airline forecasting methods, data sources, and FMSs manufactured by different companies.

The EPP only provides a representation of the active reference trajectory within the FMS based on the current active flight plan, implying that the EPP is only valid for the current clearance held by the aircraft when it is in a coupled path guidance mode (i.e., coupled Lateral Navigation (LNAV) and Vertical Navigation (VNAV)) [10]. Haugg, et al, reiterate this point, stating that it is important to consider the guidance mode, which is indicated in the EPP trajectory status portion of the message, because predictions may not be relevant when not in a coupled path guidance mode [8].

Bronsvoort, et al [10], also state that, in order to conduct accurate trajectory synchronization, as described in the concepts in Section B, a key element is missing in the EPP - an error estimate or Figure of Merit (FOM) associated 
with the EPP that informs ground automation the expected accuracy of the prediction. This information could be used by the ground system to determine the level of reliability of the EPP and thus decide whether to map the EPP directly onto a native trajectory, or to re-compute a new trajectory. The work presented in this paper attempts to address this concern through an initial characterization of this trajectory prediction error present in EPP.

\section{Analysis Design}

As stated in the previous section, the EPP message provides a set of trajectory points that are a representation of the aircraft's four-dimensional FMS reference trajectory. Under the assumption of "good FMS trajectory prediction," and without any atmospheric uncertainty (no wind), the as-flown trajectory should follow the FMS reference trajectory closely. The EPP error can be characterized against the as-flown trajectory (which includes the guidance and control error), or directly against the FMS's reference trajectory. In this analysis, we primarily focus on the EPP error against the as-flown trajectory.

The EPP message is a sampling of the FMS reference trajectory. As such, we can characterize error with respect to the reported points easily. However, characterizing error at positions between the reported points requires making assumptions about how to estimate the aircraft's position at any time between those reported points. The EPP trajectory reports on lateral trajectory change points (i.e., flight plan waypoints), vertical trajectory points (i.e., the top-of-descent and the Mach/CAS crossover altitude), and speed change points. In this analysis, we quantify the errors only at the EPP reported points. We do this for all reported points, except the speed change points.

The approach used in this analysis was to simulate a set of scenarios using a medium-fidelity simulation and compare the EPP and FMS trajectories to the as-flown trajectories.

\section{A. Simulation Environment}

NASA's Airspace and Traffic Operations Simulation (ATOS) platform was used as the simulation environment for this study. The ATOS platform contains a network of real-time simulators that can be used for batch studies and real-time human-in-the-loop experiments [15]. Each Aircraft Simulation for Traffic Operations Research (ASTOR) aircraft within the ATOS platform utilizes a high fidelity, six degree-of-freedom dynamics model, an emulated flight management system, and an emulated auto-throttle system. The ASTOR is also equipped with a pilot model that can simulate the actions of a flight crew in configuring the aircraft through climb, cruise, and descent phases of flight.

The Research Prototype Flight Management System (RPFMS) is a high-fidelity FMS simulation within ATOS with added research capabilities, such as a multiple RTA capability. The RPFMS was modified to include the ability to receive an ADS-C contract request for periodic EPP trajectory information, the ability to respond to and execute the ADS-C contract request, and the ability to encode the FMS reference trajectory into an EPP trajectory, based on the ATN-B2 specification.

The ATOS platform was used to simulate a set of ASTORs executing a set of flight plans while providing EPP trajectory reports at a pre-defined interval. Three flight plans, shown in Figure 2, were chosen to represent short, medium, and long flights across the NAS. Each flight was initiated at the start of the cruise phase of flight and flew the flight plan, through the descent phase, and terminated just prior to reaching the destination. Each flight was providing EPP trajectory information at a 60-second interval. The frequent EPP trajectory report interval provided a large amount of EPP trajectory samples while also matching the FMS trajectory update interval. The choice to begin each scenario at the start of the cruise phase of flight was made because the aircraft is not using a VNAV path guidance and is therefore not attempting to adhere to the vertical path in the EPP trajectory during the climb phase of flight. This is one limitation for the use of the EPP trajectory.

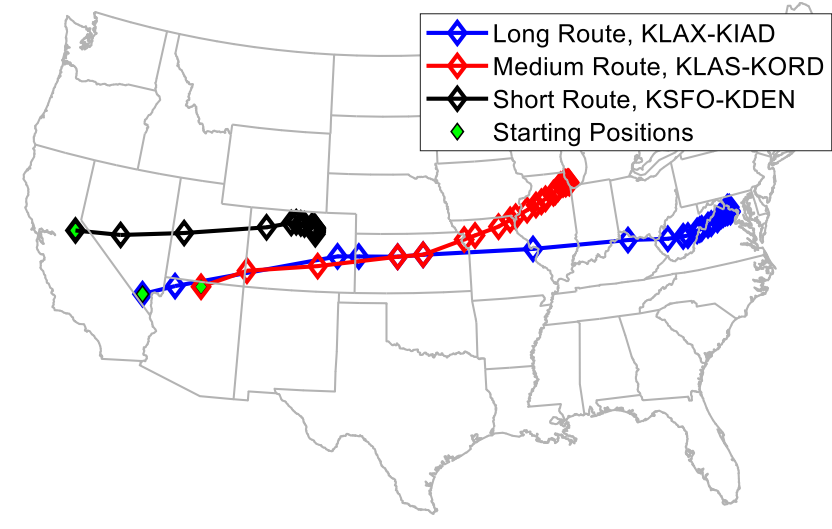

Figure 2. Long, medium, and short routes and their lateral waypoint locations. 
The ASTOR state data, FMS reference trajectory data, and EPP trajectory data were collected for each scenario. For each flight, the as-flown trajectory was gathered at a 1-Hertz frequency, a complete FMS trajectory prediction was gathered every 60 seconds during the cruise phase of flight, and a complete EPP trajectory was gathered every 60 seconds throughout the flight. These trajectories were used in post-processing to extract the EPP error information.

\section{B. Independent Variables}

The five independent variables selected to characterize the EPP error were route length, route type, wind magnitude error, wind direction error, and RTA condition. The independent variables were selected based on surveys of the literature and subject matter expertise, and directly affect the trajectory in different, quantifiable ways.

\section{Route Length and Route Type Conditions}

The two independent variables that relate to the route of flight are the route length and the route type. The route length was chosen to help identify whether longer flight plans have the same or different EPP error characteristics when compared to shorter flight plans. The route type was used to distinguish between a full route and a sparse route, where a sparse route will have significantly fewer flight plan waypoints than a full route, and may have different EPP error characteristics. Additional details about each route of the three routes simulated in this analysis can be found in Table 1 and Table 2 .

Table 1. Route descriptions - all route lengths.

\begin{tabular}{ccccc}
\hline $\begin{array}{c}\text { Route } \\
\text { Length }\end{array}$ & $\begin{array}{c}\text { Departure } \\
\text { Airport }\end{array}$ & $\begin{array}{c}\text { Initializatio } \\
\text { n Point }\end{array}$ & $\begin{array}{c}\text { Cruise } \\
\text { Altitude }\end{array}$ & $\begin{array}{c}\text { Arrival } \\
\text { Airport }\end{array}$ \\
\hline \hline $\begin{array}{c}\text { Long } \\
\text { (RL1) }\end{array}$ & KLAX & $\begin{array}{c}35.9936^{\circ} \mathrm{N} \\
115.2879^{\circ} \mathrm{W}\end{array}$ & FL390 & KIAD \\
Medium & KLAS & $\begin{array}{c}36.7596^{\circ} \mathrm{N} \\
111.7164^{\circ} \mathrm{W}\end{array}$ & FL350 & KORD \\
(RL2) & & & \\
Short & KSFO & $\begin{array}{c}38.4483^{\circ} \mathrm{N} \\
120.2139^{\circ} \mathrm{W}\end{array}$ & FL350 & KDEN \\
(RL3) & & & & \\
\hline
\end{tabular}

Table 2. Route waypoints - all route lengths, full and sparse route types.

\begin{tabular}{|c|c|c|c|c|}
\hline $\begin{array}{l}\text { Route } \\
\text { Length }\end{array}$ & $\begin{array}{l}\text { En Route Flight } \\
\text { Plan (Full } \\
\text { Route/RT1) }\end{array}$ & $\begin{array}{l}\text { En Route Flight } \\
\text { Plan (Sparse } \\
\text { Route/RT2) }\end{array}$ & Arrival Route & Approach \\
\hline $\begin{array}{l}\text { Long } \\
\text { (RL1) }\end{array}$ & $\begin{array}{l}\text { NOOTN, SELLS, } \\
\text { OATHE, HYS, SLN, } \\
\text { STL, FLM, HVQ }\end{array}$ & NOOTN, HVQ & $\begin{array}{l}\text { HVQ, FIMPA, WOJOW², BURTT, PHOOW, } \\
\text { HESEE }^{2,3,4}, \text { GYSLO }^{3,4}, \text { BBONE }^{3,4}, \text { KILMRR }^{2,3,4} \text {, } \\
\text { OTTTO }^{4}, \text { MAAAY }^{4}, \text { RYPIN, GIBBZ, SUNY, }^{\text {UDIYU, MATTC }} \\
\text { UDIYU }\end{array}$ & IZUMI, DOMSE, R-19L \\
\hline $\begin{array}{l}\text { Medium } \\
\text { (RL2) }\end{array}$ & $\begin{array}{l}\text { DVC, PUB, HYS, } \\
\text { SLN, AGENT, } \\
\text { KIDER, IRK }\end{array}$ & DVC, IRK & 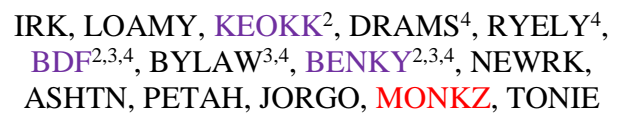 & $\begin{array}{l}\text { WAVIE, ADAMIE, } \\
\text { WILLT } \\
\text { FIDAK, R-28R }\end{array}$ \\
\hline $\begin{array}{l}\text { Short } \\
\text { (RL3) }\end{array}$ & $\begin{array}{l}\text { INSLO, GAROT, } \\
\text { EKR }\end{array}$ & INSLO, EKR & $\begin{array}{l}\text { EKR }^{2}, \mathrm{CSPAD}^{4}, \mathrm{FRNCH}^{4}, \mathrm{SKARF}^{4}, \\
\text { TOMSN }^{2,3,4}, \mathrm{BEOND}^{3,4}, \mathrm{SWAYN}^{4}, \mathrm{KAILE}^{2,3,4}\end{array}$ & $\begin{array}{l}\text { BJETN }^{4}, \text { JEEPR }^{4}, \\
\text { JOBOB }^{4}, \text { KUURT }^{4}, \\
\text { KIKME }^{4}, \text { LEETS }^{4}, \text { R-16L }\end{array}$ \\
\hline
\end{tabular}

The long, medium, and short flight routes (depicted in Figure 2) are represented by the labels RL1, RL2, and RL3, respectively. The route type was chosen to be either a full route (i.e., a route similar to what an aircraft would fly on a normal day), designated by condition RT1, or a sparse route (i.e., a route with a minimal set of waypoints in the cruise phase of flight), designated by condition RT2. The selected routes were designed to not have discontinuities and to travel in the same general west-to-east direction so that one scenario could contain three aircraft flying in the same wind field. The three routes were based on historical flights of a Boeing 757 aircraft.

\section{Wind Conditions}

Wind forecast error is widely known to be a significant contributor to trajectory prediction error. In this study, nine wind conditions were chosen to demonstrate the impact of both wind forecast magnitude error and wind forecast direction error. The wind direction component was held constant (i.e., wind from 080 degrees everywhere) within

\footnotetext{
${ }^{2}$ Waypoints with RTA constraints in some scenario runs.

${ }^{3}$ Analysis waypoint with an associated speed constraint.

${ }^{4}$ Analysis waypoint with an associated altitude constraint.
} 
each scenario, while the wind magnitude, which increased from the ground level to 40,000 feet, was modeled using a linear fit to ensemble recorded wind data. Within each scenario, there was a constant truth wind field (the wind experienced by the aircraft dynamics) and a constant forecast wind field (the wind the FMS used for wind and trajectory predictions).

Wind magnitude error was implemented by using wind forecast magnitude equal to truth magnitude, forecast magnitude greater than truth magnitude, and forecast magnitude smaller than truth magnitude. The wind magnitude conditions were described as the percentile model fit to the ensemble recorded data. These were $25^{\text {th }}$-percentile, $50^{\text {th }}$ percentile, and $75^{\text {th }}$-percentile wind magnitude models, with the $50^{\text {th }}$-percentile model chosen as the truth wind magnitude [16]. As an example, this $50^{\text {th }}$ percentile model had a wind magnitude varying linearly from approximately 10 knots at 5,000 feet to approximately 50 knots at 40,000 feet.

Wind direction error was implemented with a transition from a nearly pure headwind condition to a nearly pure tailwind condition, with wind directions of 080, 110, 140, 170, 215, and 260 degrees across different scenarios. These translate to a wind direction forecast error of 0,30 , $60,90,135$, and 180 degrees when compared to the truth direction of 080 degrees.

The nine wind conditions used in this analysis are presented in Table 3. The wind magnitude and direction conditions are also shown in Figure 3.
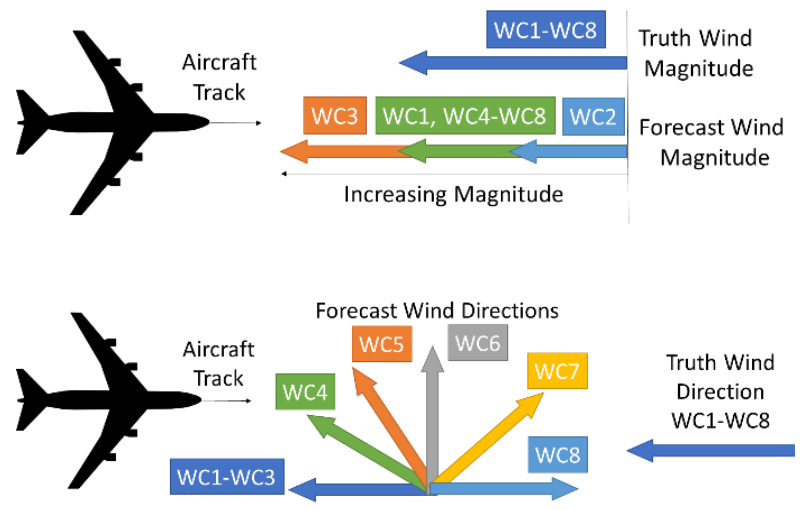

Figure 3. Wind magnitude conditions (top) and wind direction conditions (bottom).

Table 3. Scenario wind conditions.

\begin{tabular}{cccccc}
\hline $\begin{array}{c}\text { Condition } \\
\text { Number }\end{array}$ & Qualifier & $\begin{array}{c}\text { Truth } \\
\text { Wind } \\
\text { Magnitude } \\
\text { Percentile }\end{array}$ & $\begin{array}{c}\text { Truth Wind } \\
\text { Direction }\end{array}$ & $\begin{array}{c}\text { Forecast } \\
\text { Wind } \\
\text { Magnitude } \\
\text { Percentile }\end{array}$ & $\begin{array}{c}\text { Forecast } \\
\text { Wind } \\
\text { Direction }\end{array}$ \\
\hline \hline WC0 & No Wind & N/A & N/A & N/A & N/A \\
WC1 & Perfect Knowledge (Truth = Forecast) & $50^{\text {th }}$ & From $080^{\circ}$ & $50^{\text {th }}$ & From $080^{\circ}$ \\
\hline WC2 & Positive Magnitude Error (Truth > Forecast) & $50^{\text {th }}$ & From $080^{\circ}$ & $25^{\text {th }}$ & From $080^{\circ}$ \\
\hline WC3 & Negative Magnitude Error (Truth < Forecast) & $50^{\text {th }}$ & From $080^{\circ}$ & $75^{\text {th }}$ & From $080^{\circ}$ \\
\hline WC4 & $30^{\circ}$ Direction Error & $50^{\text {th }}$ & From $080^{\circ}$ & $50^{\text {th }}$ & From $110^{\circ}$ \\
\hline WC5 & $60^{\circ}$ Direction Error & $50^{\text {th }}$ & From $080^{\circ}$ & $50^{\text {th }}$ & From $140^{\circ}$ \\
\hline WC6 & $90^{\circ}$ Direction Error & $50^{\text {th }}$ & From $080^{\circ}$ & $50^{\text {th }}$ & From $170^{\circ}$ \\
\hline WC7 & $135^{\circ}$ Direction Error & $50^{\text {th }}$ & From $080^{\circ}$ & $50^{\text {th }}$ & From $215^{\circ}$ \\
WC8 & $180^{\circ}$ Direction Error & $50^{\text {th }}$ & From $080^{\circ}$ & $50^{\text {th }}$ & From $260^{\circ}$ \\
\hline
\end{tabular}

\section{RTA Conditions}

The use of an RTA waypoint on a route was expected to have an impact on the EPP error characteristics. In order to understand this impact, four RTA conditions were tested. The first condition (No RTA) involved no RTA on the route. The second condition (RTA1) involved an RTA waypoint near the end of the cruise segment for each flight. In this condition, an RTA was assigned to the waypoints WOJOW, KEOKK, and EKR of the long, medium, and short routes, respectively. The third condition (RTA2) involved an RTA in the descent portion of the flight, just after the top-of-descent point. In this condition, an RTA was assigned to the waypoints HESEE, BDF, and TOMSN of the long, medium, and short routes, respectively. The fourth condition (RTA3) involved an RTA to a waypoint at the 
entry to the terminal area (defined here as a point just prior to reaching 10,000 feet). In this condition, an RTA was assigned to the waypoints KILMR, BENKY, and KAILE of the long, medium, and short routes, respectively.

In each RTA condition, an RTA tolerance of $+/-30$ seconds was used. It is important to note that the RPFMS used in this analysis has dynamic RTA tolerances that can result in internal tolerances of up to 120 seconds when far from the RTA waypoint and 30-second tolerance as the flight approaches the RTA point.

\section{Test Scenarios}

A select combination of the conditions of each independent variable above resulted in the experiment matrix shown in Table 4. The experiment matrix consists of 13 scenario runs, each consisting of one long, one medium, and one short route length flight simulated simultaneously. That equates to 39 flight datasets of asflown trajectories, FMS trajectories, and EPP trajectories.

Each scenario in Table 4 resulted in three flown trajectories and multiple EPP and FMS trajectories. As an example of the output data of each scenario, scenario 1 resulted in 219, 135, and 84 FMS trajectories for the long, medium, and short routes, respectively. Scenario 1 also had 236, 158, and 102 EPP trajectories for those same three flights. The number of EPP trajectories for each flight, in general, exceeded the number of FMS trajectories because the FMS trajectories in the RPFMS were not updated after top-of-descent but the EPP trajectories continued to be generated using the last Table 4. Test matrix.

\begin{tabular}{ccccc}
\hline Scenario & Route Length & $\begin{array}{c}\text { Route } \\
\text { Type }\end{array}$ & Wind & RTA \\
\hline \hline 1 & RL1, RL2, RL3 & RT1 & WC0 & No RTA \\
2 & RL1, RL2, RL3 & RT1 & WC1 & No RTA \\
3 & RL1, RL2, RL3 & RT1 & WC2 & No RTA \\
4 & RL1, RL2, RL3 & RT2 & WC2 & No RTA \\
5 & RL1, RL2, RL3 & RT1 & WC3 & No RTA \\
6 & RL1, RL2, RL3 & RT1 & WC4 & No RTA \\
7 & RL1, RL2, RL3 & RT1 & WC5 & No RTA \\
8 & RL1, RL2, RL3 & RT1 & WC6 & No RTA \\
9 & RL1, RL2, RL3 & RT1 & WC7 & No RTA \\
10 & RL1, RL2, RL3 & RT1 & WC8 & No RTA \\
11 & RL1, RL2, RL3 & RT1 & WC2 & RTA1 \\
12 & RL1, RL2, RL3 & RT1 & WC2 & RTA2 \\
13 & RL1, RL2, RL3 & RT1 & WC2 & RTA3 \\
\hline
\end{tabular}
FMS reference trajectory.

\section{Dependent Variables}

The dependent variables for this EPP error analysis were the cross-track error, vertical error, and time error. The three dependent variables were computed with respect to the state data point closest to the trajectory reported point. This closest state data point was determined with respect to the closest lateral position, in the latitude-longitude plane, to the EPP reported latitude and longitude. For a reported fly-by waypoint, the middle-of-turn (MOT) point position was estimated using the EPP-reported radius and the track angle change between subsequent points. The cross-track error was measured by the Great Circle distance between the closest state position and the EPP reported position along a line tangent to the EPP trajectory's track at the reported point (taking into account any track change at fly-by waypoints). The cross-track error was always positive because no distinction was made between left-of-track or rightof-track errors. The vertical error was measured as the difference between the closest state data point's altitude and the EPP reported altitude. Positive vertical error indicates that the aircraft sequenced the reported point above the reported altitude. Similarly, the time error was measured as the difference between the closest state data point's time and the EPP reported time, where positive error indicates the aircraft sequenced the reported point after the reports time (late).

We note that the estimation of the MOT point for the EPP trajectory can be problematic. The track in and out of a fly-by waypoint (i.e., to compute the track angle change) must be estimated given the other points provided in the EPP trajectory because the EPP trajectory does not directly provide track information. One area where this is problematic is for the first point in the EPP trajectory, which, in general, is a point somewhere ahead of the aircraft's current position. Thus, the track into this first EPP point must be assumed to be from the current aircraft position, which may not necessarily be an accurate assumption. Note that, in the case of the FMS trajectory from the RPFMS, the MOT point is available and does not need to be re-computed for this error analysis.

\section{Results and Analysis}

In this section, a preliminary characterization of EPP trajectory errors is presented. The characterization is a qualitative discussion of the EPP trajectory errors observed in the simulated scenarios. In many of the figures and 
discussion of this paper, the EPP error metrics are presented as a function of the time horizon. The time horizon refers to the difference between the estimated time-of-arrival at a waypoint and the time the trajectory was generated. Some may also refer to this as the "time-to-go" to that waypoint at trajectory generation time.

\section{A. EPP and FMS Comparison - No Wind}

The baseline scenario with zero wind magnitude (scenario 1) allows for the comparison of the EPP error data against the FMS error data. In this scenario, the FMS error represents the combination of the trajectory prediction error and the guidance error in following the desired flight plan. The EPP error is a combination of this FMS error, the error associated with transforming the FMS reference trajectory into the EPP format, the error associated with computing the geometric middle-of-turn, and the error that results from the EPP trajectory itself being "stale." Staleness here refers to an EPP message that may not be a representation of the most recent FMS reference trajectory.

The FMS cross-track error is a function of the error in the prediction of the MOT point for a fly-by waypoint combined with the fact that the MOT point is, itself, not a point that the guidance algorithm is targeting. In some cases, such as with very small track angle changes ( 1-2 degrees or smaller), the FMS will not compute a turn radius or MOT point, which results in cross-track error at those points. The EPP, because it is generated from the FMS reference trajectory, necessarily inherits this guidance and prediction error. In addition to these errors, the EPP, in general, will have higher cross-track errors because the EPP message does not contain the FMS's estimated MOT point. The EPP message also limits the resolution of this reported fly-by radius to the nearest $1 / 10^{\text {th }}$ of a NM, thereby creating additional possibility for errors.

Figure 4 shows the EPP cross-track error for the no wind baseline run as a function of the time horizon. Figure 5 shows the FMS cross-track error for the same run. The figures show the EPP or FMS cross-track errors for each point of each EPP or FMS reference trajectory, respectively, for each route length of the baseline run. The largest value of time horizon for any given point's error profile is representative of how far in time that point is in the trajectory relative to the scenario starting position. Both figures indicate a steady cross-track error for each waypoint throughout the scenario run, which is expected when there is no wind error affecting the trajectory predictions of the FMS. However, the scale of the cross-track error does show a larger cross-track error for some of the EPP points as compared to the same points in the FMS trajectory. This is due to the MOT estimation and the resolution of the EPP reported turn radius. We note that the error profiles for most points are relatively constant at all time horizons, indicating that the FMS trajectory predictions are also relatively constant. Consequently, the non-zero steady state error at zero time horizon comes from guidance error and EPP trajectory inferences.

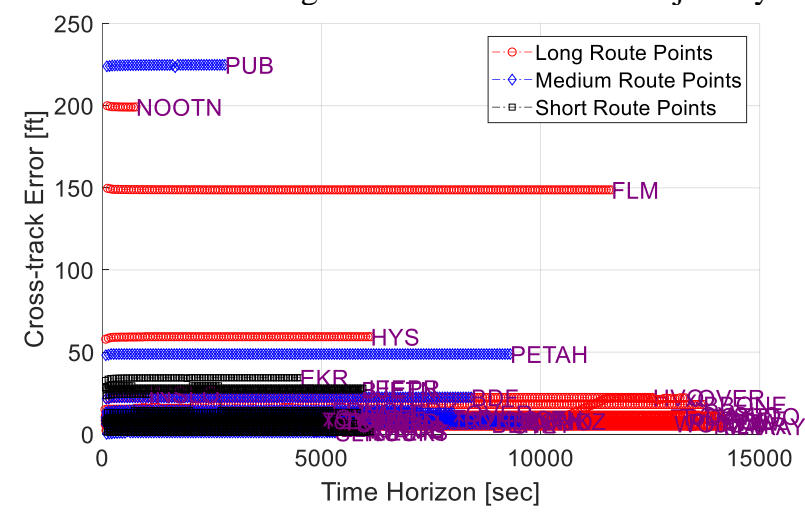

Figure 4. EPP cross-track errors as a function of the time horizon [RL1-RL3, RT1, WC0, No RTA].

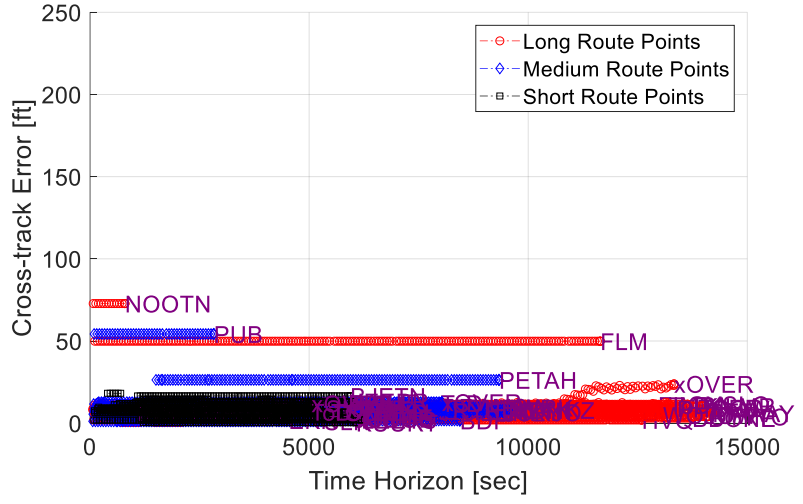

Figure 5. FMS cross-track errors as a function of the time horizon [RL1-RL3, RT1, WC0, No RTA].

The vertical error is the difference between the actual crossing altitude at a point and the predicted altitude in each EPP or FMS trajectory. Figure 6 shows the vertical error for the EPP trajectory points versus time horizon for scenario 1 while Figure 7 shows the vertical error for the same FMS trajectory points. While the two datasets show similar characteristics, there are two distinguishing features when comparing these two figures. First, the EPP error data has trajectory errors all the way down to zero time horizon for all points whereas the FMS error data terminates just prior to zero time horizon for some of the trajectory points. This is because, in the prototype FMS used in this analysis, the FMS reference trajectory is not re-computed in the descent phase of flight (the last reference trajectory is computed 
prior to top-of-descent) while that same FMS reference trajectory is used to generate EPP trajectory reports in the descent phase. Second, there is some level of "noise" in the EPP error data, which is attributed to truncation or rounding of the reported altitude to increments of 10 feet.

The vertical error profiles of both the EPP and FMS show relatively constant error profiles with the exception of a few points. The points BURTT, ToD, and xOVER in the long route show some variation in their vertical error with time horizon. This variation is due to re-computations and updates to the descent profile as the flight progresses, where the inflection points in the error data represent the sequencing of a flight plan waypoint. Note also that BURTT happens sequentially after the ToD and before the xOVER points in the flight, and that the ToD and xOVER points have dynamically generated positions based on the descent profile computations, which explains why the predicted altitude at BURTT is changing. The ToD point has a vertical error of $\sim 150-200$ feet, which is a direct result of the way this point is defined in both the EPP trajectory and the FMS trajectory. The ToD point is defined by the intersection of the cruise altitude with the descent path. The implication of this definition is that the aircraft has already begun intercepting the descent path as it sequences the predicted latitude and longitude position of the ToD point, leading to a below-path (negative) vertical error.

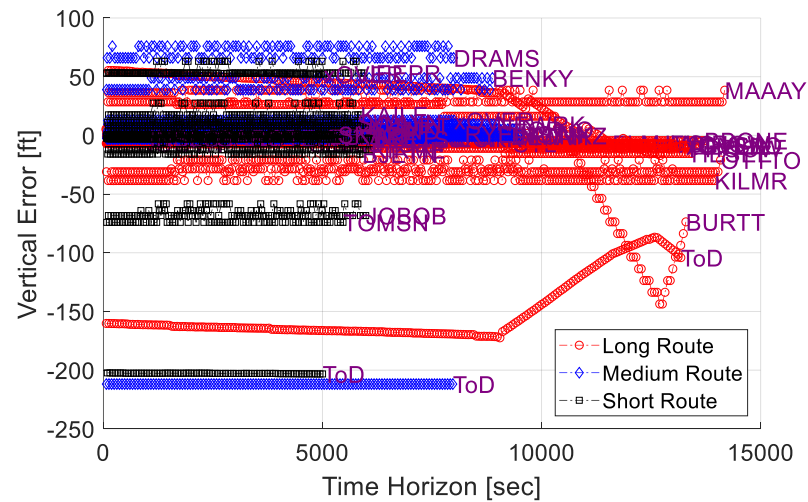

Figure 6. EPP vertical errors as a function of the time horizon [RL1-RL3, RT1, WC0, No RTA].

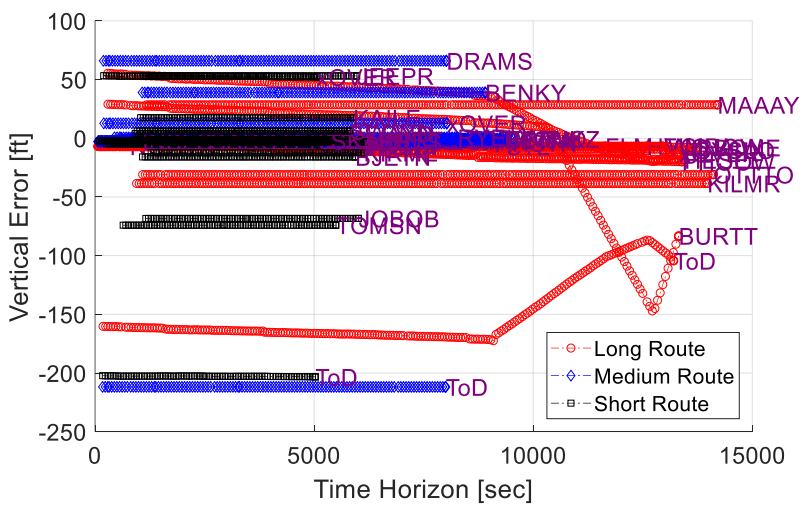

Figure 7. FMS vertical errors as a function of the time horizon [RL1-RL3, RT1, WC0, No RTA].

The time or temporal error is the difference between the actual crossing time at a point and the predicted time in each EPP or FMS trajectory. Figure 8 shows the temporal error for the EPP trajectory points versus time horizon for scenario 1 while Figure 9 shows the temporal error for the same FMS trajectory points. There are two characteristic differences between the EPP and FMS temporal errors. First, the EPP temporal errors exhibit a "stair-stepping" pattern when compared to the FMS temporal errors. This is the result of the resolution of the time component in the EPP

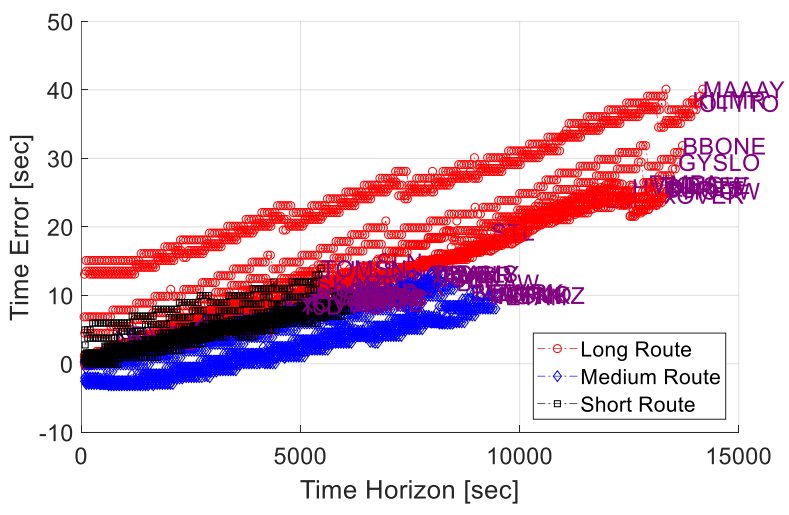

Figure 8. EPP time errors as a function of the time horizon [RL1-RL3, RT1, WC0, No RTA].

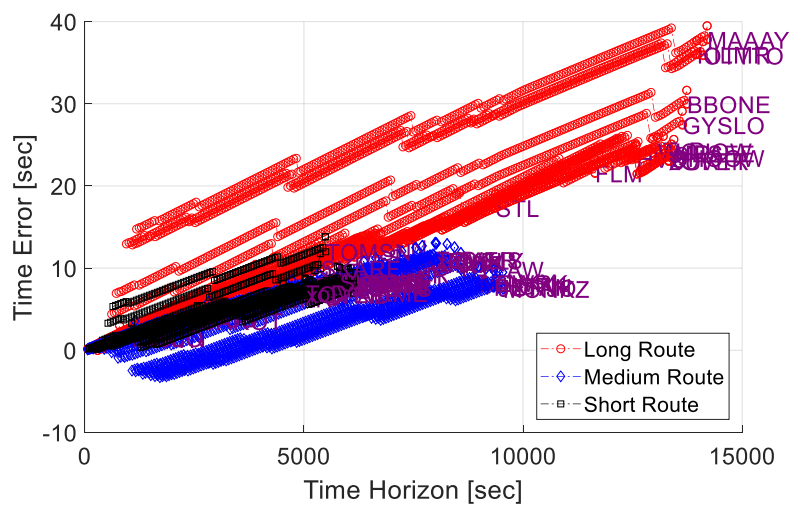

Figure 9. FMS time errors as a function of the time horizon [RL1-RL3, RT1, WC0, No RTA].

trajectory points (1-second resolution). The RPFMS reported trajectory points were provided as floating point numbers with a ten-millisecond resolution. Second, as discussed previously, FMS trajectories are not re-computed 
beyond the top-of-descent point so the error data appears to end prior to zero time horizon for some points. This lack of ETA updates beyond the top-of-descent point is also an artifact of the RPFMS simulation and may not be consistent with how operational FMSs handle trajectory updates.

The FMS error data shows discontinuities, or "jumps," in the temporal error data at different points in the time horizon. These discontinuities represent a new FMS trajectory prediction (typically associated with the sequencing of a waypoint) where the RPFMS re-computes the FMS trajectory instead of simply updating ETA information. The linear decrease in the temporal error as the time horizon decreases represents the FMS trajectory prediction error that is reduced as the aircraft gets closer to sequencing a trajectory point. In general, this particular FMS and aircraft simulation combination had trajectory predictions that were biased towards earlier than actual waypoint crossing predicted times; other FMS/aircraft combinations may exhibit the opposite behavior. Trajectory points with temporal error that does not converge to zero error represent points in the descent phase of flight where the FMS trajectory is not being updated.

\section{B. No Wind versus Perfect Wind}

The comparison of scenario 1 (no wind) with scenario 2 (perfect wind), is intended to highlight any differences in the EPP and FMS trajectories in the presence of a non-zero wind field. This comparison is important because the FMS only samples the forecast wind at discrete points of the flight plan (namely, at the flight plan waypoints and at a few discrete altitudes for the descent phase). In addition, the FMS uses wind blending (where the aircraft-sensed wind information is blended with the upstream forecast wind) to compute wind predictions for waypoints within some distance ahead of the aircraft's current position. As such, the FMS trajectories will have some non-zero prediction error that results from this sampling and blending of the wind information that will affect the EPP trajectory data.

Figure 10 shows the EPP cross-track error for scenario 2 as a function of the time horizon and can be compared to Figure 4 for scenario 1. The EPP median cross-track errors for scenario 2 and scenario 1 are shown in Figure 11 for the long route points (medium and short routes, not shown here, show similar characteristics). The EPP errors are smaller in scenario 2 than in scenario 1 for most trajectory points. For example, the waypoint NOOTN has approximately 120 feet cross-track error in the perfect wind scenario as compared to approximately 200 feet in the no wind scenario. The smaller cross-track errors appear to be a result of the smaller turn radii predicted in the perfect wind scenario. In this scenario, the wind is nearly pure headwind, which results in smaller groundspeed for the same airspeed and requires a smaller turn radius for a given turn. Because of the FMS guidance algorithms, there exist some turn radii that produce better cross-track performance (e.g., the executed turn is the closest to the predicted turn) with respect to the FMS predictions. For the perfect wind scenario in the presence of a headwind, this minimum crosstrack error occurs for points with predicted fly-by-radius around 40 nautical miles; points with other turn radii have larger cross-track errors.

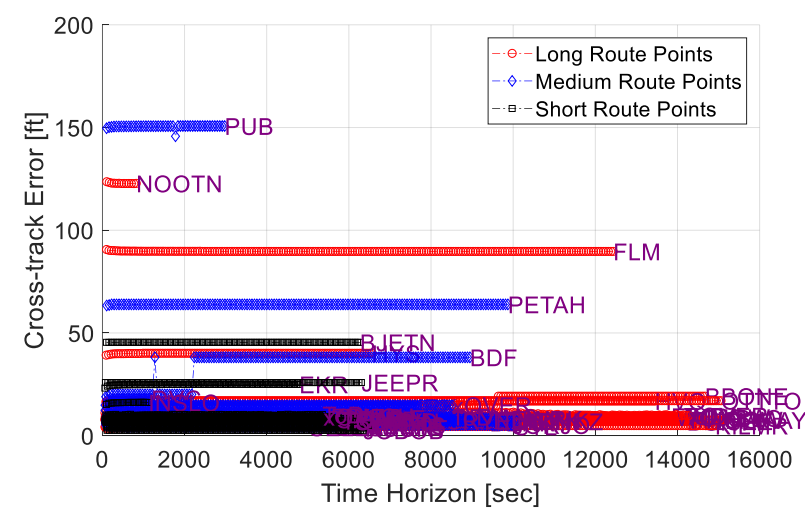

Figure 10. EPP cross-track errors as a function of the time horizon [RL1-RL3, RT1, WC1, No RTA].

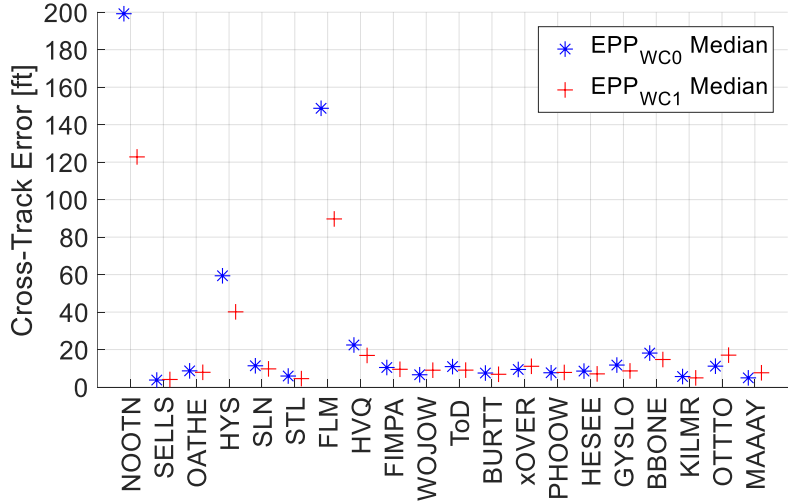

Figure 11. No wind $\left(\mathrm{EPP}_{\mathrm{wc} 0}\right)$ and perfect wind (EPPwC1) EPP median cross-track error comparison for the long route [RL1, RT1, No RTA].

Figure 12 shows the vertical error for the EPP trajectory points versus time horizon for scenario 2 and can be compared to Figure 6 for scenario 1. The EPP median vertical errors for scenario 2 and scenario 1 are shown in Figure 13 for the long route points. Most of the vertical errors are close to zero, or within 20 feet, with the exception of ToD, 
BURTT, $x O V E R$, KILMR, OTTTO, and MAAAY on the long route. The ToD point has the worst-case vertical error (nearly 200 feet) because of the EPP definition of this point as the intersection point between the descent path and the en route path. The other five points have median vertical errors under 50 feet and are, generally, the points on the vertical profile not affected by an altitude constraint as the FMS trajectory prediction changes.

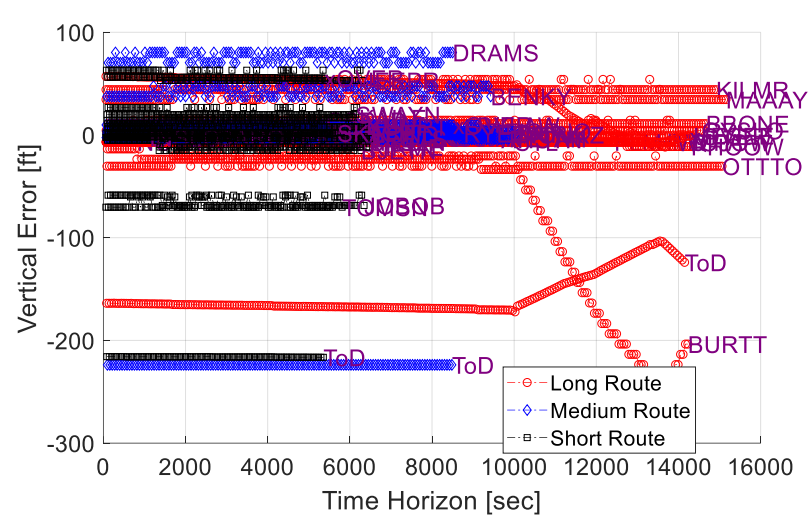

Figure 12. EPP vertical errors as a function of the time horizon [RL1-RL3, RT1, WC1, No RTA].

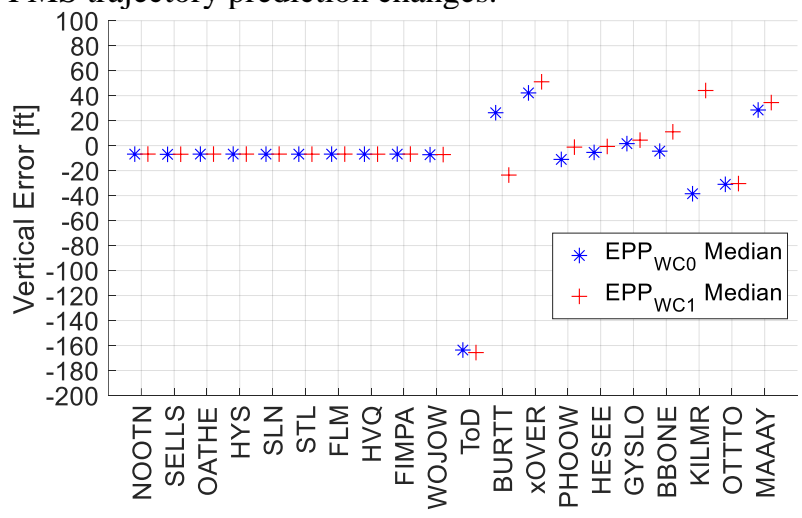

Figure 13. No wind (EPPwco) and perfect wind (EPPwC1) EPP median vertical error comparison for the long route [RL1, RT1, No RTA].

The EPP temporal errors for scenario 1 and 2 were binned by time horizon and are shown in Figure 14 as the means and standard deviations for each bin. The temporal error data shows smaller standard deviations for the scenario with perfect wind (scenario 2) as compared to the scenario with no wind (scenario 1), but the differences in the temporal error curves are not statistically significant. The smaller standard deviations indicate only slightly better trajectory predictions in the presence of the wind field, which could be considered purely coincidental.

\section{Impact of Sparse Versus Full Routes}

In this section, the differences between the full routes scenario (scenario 3 ) and the sparse route scenario (scenario 4) are compared. Note that the scenarios were both run in the presence of a true

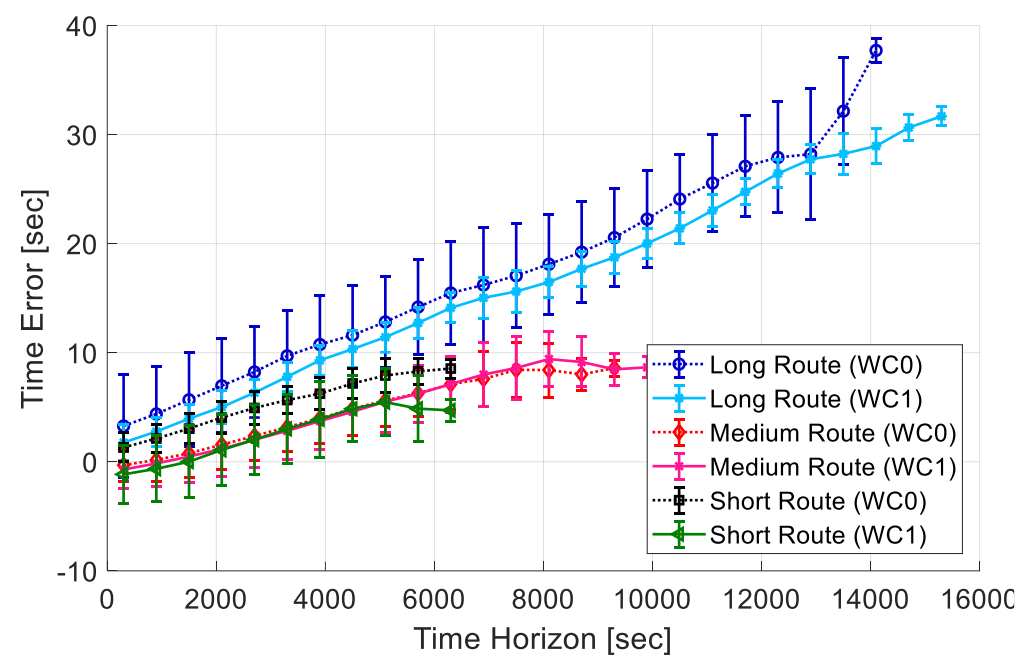

Figure 14. Binned EPP temporal error mean and standard deviation for all trajectory points in the no wind [RL1-RL3, RT1, WC0, No RTA] and the perfect wind [RL1-RL3, RT1, WC1, No RTA] scenarios.

wind with magnitude greater than the forecast magnitude (WC2) in order to assess the impact of the forecast winds on a flight plan with long legs (e.g., the sparse flight routes). Any significant differences in EPP error profile will manifest themselves in the cruise phase of the flight because the descent portion of both scenarios is identical in terms of the flight plan waypoints.

Figure 15 shows the median cross-track errors for the waypoints common to both the full routes (RT1) and the sparse routes (RT2) scenarios for the long route (medium and short routes show similar characteristics). The median cross-track error difference between the two scenarios appears to be the largest at the waypoint NOOTN. A closer look at the data revealed that the difference was linked to different turn radii at the point NOOTN resulting from different track angle change to go direct to HVQ in the sparse routes scenario ( $\sim 7$ degrees) versus the track angle 
change to go directly to PUB in the full routes scenario ( $\sim 3$ degrees). A similar phenomenon with large median error differences occurs at the point HVQ, the effective end of the sparse en route portion of the flight plan. All other points on the long route have a median error difference less than 15 feet cross-track error. Qualitatively, with the exception of the start and end waypoints of the sparse routes, the cross-track error profiles of the full and sparse routes are similar.

Qualitatively, there is little difference between EPP vertical errors of the full route scenario (scenario 3 ) as compared to the sparse routes scenario (scenario 4). Figure 16 shows the vertical error for the points of the sparse routes scenario. We do note, however, the discontinuity that appears in the vertical error of some of the route points, both on the full routes and the sparse routes. This discontinuity is due to the piece-wise linear FMS wind blending function, which triggered a significant update to the vertical profile as the wind predictions changed when the flight sequenced one of the route's en route waypoints. The points with the large discontinuity in vertical error are those on the descent profile without any hard "AT" altitude constraints, where an update to the predicted winds can have a significant impact on the predicted crossing altitudes. These types of error discontinuities are governed by the algorithms that each FMS uses to convert a wind forecast into a set

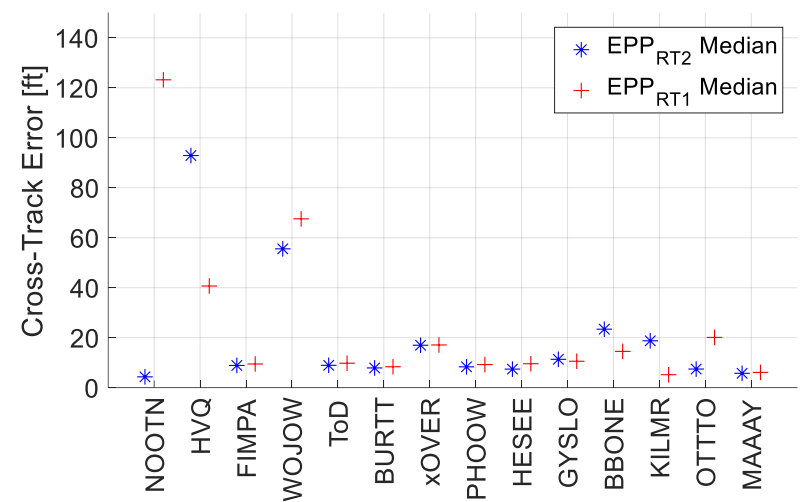

Figure 15. Sparse route (EPP $\left.\mathbf{R T 2}_{2}\right)$ and full route (EPP $\left._{\mathrm{RT1}}\right)$ median EPP cross-track errors for the trajectory points of the long route [RL1, WC2, No RTA]. of wind predictions along the route.

Figure 17 shows the median vertical errors for the waypoints common to both the full and the sparse routes scenarios for the long route. In all instances, the vertical error profiles are the same and the median vertical error difference is within 10 feet, which is the EPP reported altitude resolution. This is to be expected because the primary difference between the full and the sparse routes is in the en route phase of flight where the aircraft is level at the cruise altitude and no significant difference would be expected in the EPP reported altitudes. The large mean vertical errors do point to the fact that the FMS trajectory predictions may have large errors that will be reflected in the EPP until the aircraft enters the wind blending region and the FMS prediction has the opportunity to reduce the vertical error, as evidenced at short time horizons in Figure 16.

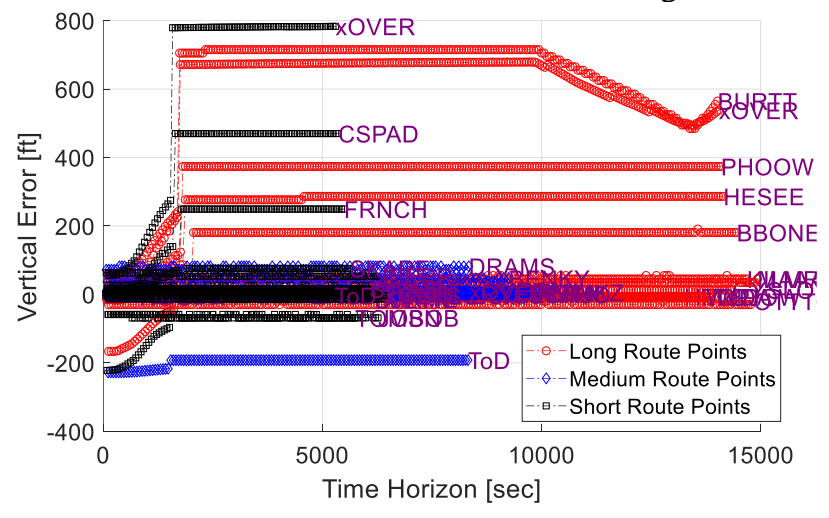

Figure 16. Vertical error for EPP trajectory points as a function of the time horizon for the sparse routes scenario [RL1-RL3, RT2, WC2, No RTA].

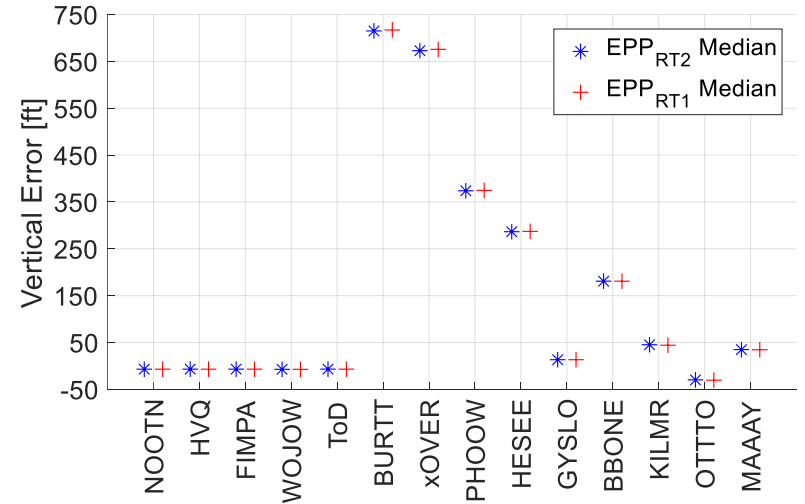

Figure 17. Sparse routes $\left(\mathrm{EPP}_{\mathrm{RT} 2}\right)$ and full routes (EPPRT1) EPP median vertical error for the trajectory points of the long route [RL1, WC2, No RTA].

Figure 18 shows the binned mean and standard deviation of the time error for all route points of each route length and route type as a function of the time horizon. Qualitatively, there are observed differences between the two temporal error profiles, particularly with respect to the magnitude and slope of the time error above approximately 5000 seconds of time horizon. The time error differences between the full routes and the sparse routes are the 
combined result of two different aspects of trajectory prediction used by the FMS. First, the FMS only implements wind information at discrete waypoints. As such, the sparse routes have less en route waypoints and, thus, less wind information. For example, in the sparse long route, the FMS has wind information for the waypoints NOOTN and HVQ, which are approximately 1500 nautical miles apart, whereas, in the full long route, the FMS has wind information at six additional waypoints between NOOTN and HVQ. The second aspect of FMS trajectory prediction that contributes to the time error differences is the wind blending. As described before, the wind blending blends the true winds with the forecast winds out to approximately 700 nautical miles ahead of the aircraft, where, at that horizon, the predicted winds are equal to the forecast winds. In the case of

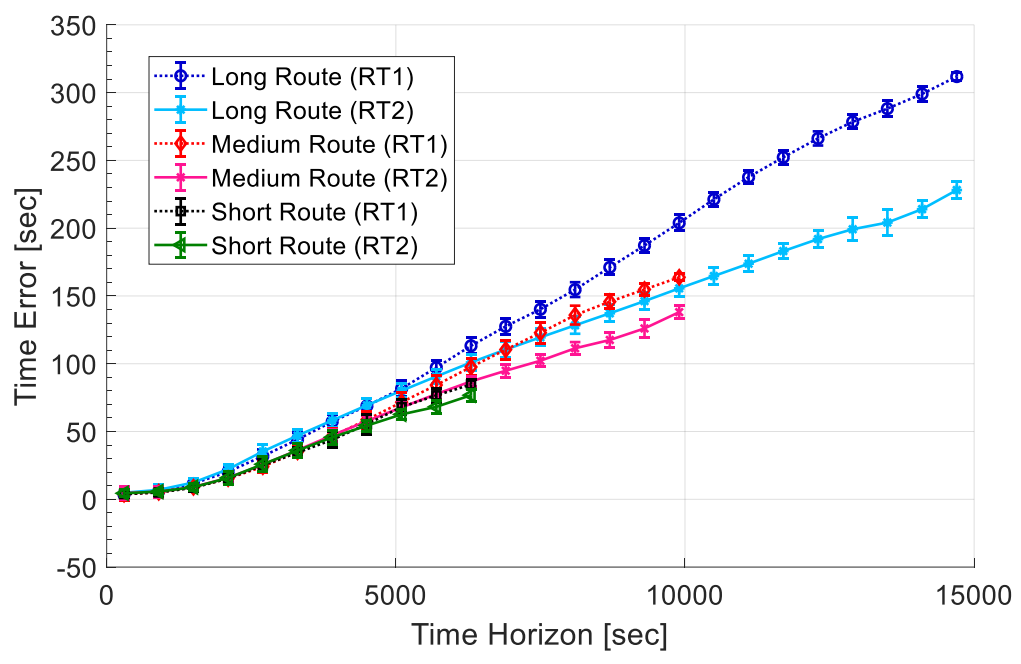

Figure 18. Binned temporal error mean and standard deviation for all trajectory points in the full routes scenario [RL1-RL3, RT1, WC2, No RTA] and the sparse routes scenario [RL1-RL3, RT2, WC2, No RTA].

the sparse routes, there are less opportunities for the wind blending to include wind forecast errors in the wind predictions during the long route legs. This explains why the time error profiles converge at or below approximately 5000 seconds time horizon as this horizon is where the impact of wind blending begins to include wind forecast data that may exist for the scenarios we have tested here.

\section{Wind Magnitude Error Effects}

Wind magnitude error effects were investigated using a set of three scenarios. These scenarios represent the conditions where: the true wind magnitude was equal to the forecast wind magnitude (scenario 2), the true wind magnitude was larger than the forecast wind magnitude (scenario 3), and the true wind magnitude was smaller than the forecast wind magnitude (scenario 5). In all scenarios, the true and predicted wind direction was from 080 degrees, or nearly pure headwind.

Figure 19 shows the cross-track errors for all route lengths and all route points of the three wind magnitude conditions tested: truth equal to forecast (WC1), truth greater than forecast (WC2), and truth less than forecast (WC3) [RL1-RL3, RT1, No RTA]. The cross-track errors from these three wind conditions indicate nearly symmetrical error profiles with respect to the perfect wind conditions. For example, at long time horizons, the EPP reported waypoint FLM has approximately 90 feet cross-track error in the perfect wind condition (WC1), approximately 101 feet cross-track error in the under-forecast wind magnitude case (WC2), and

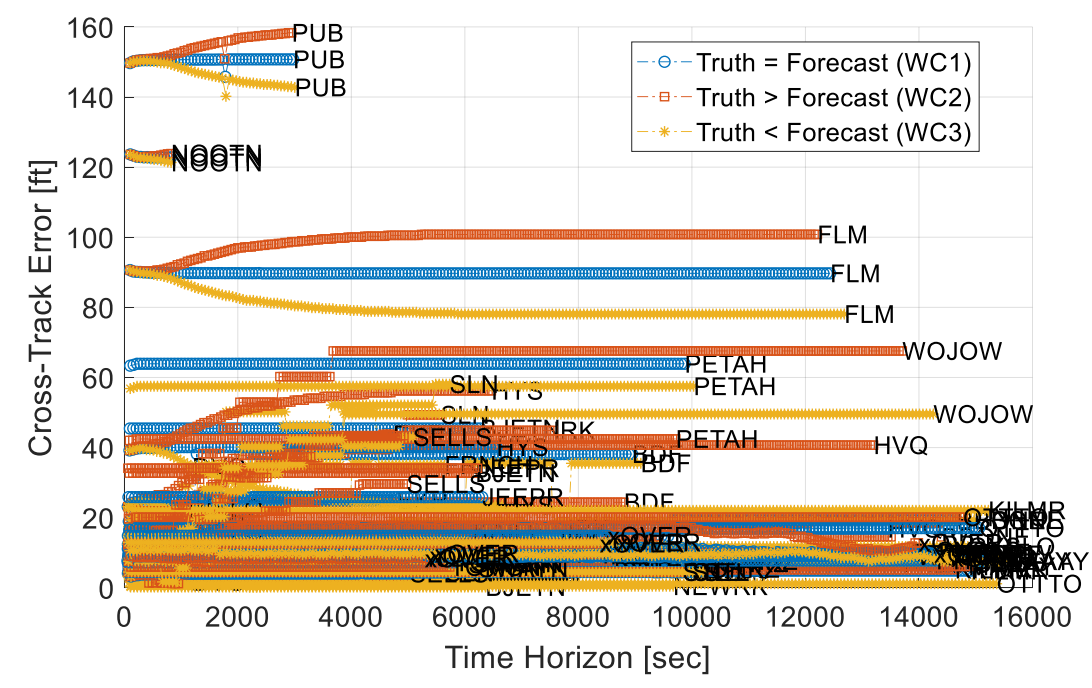

Figure 19. EPP cross-track error for the perfect wind condition (WC1), the true wind magnitude greater than forecast (WC2), and the true wind magnitude less than forecast (WC3) [RL1-RL3, RT1, No RTA]. 
approximately 78 feet cross-track error in the over-forecast wind magnitude case (WC3). At zero time horizon, these cross-track errors all converge to 96.3 feet cross-track error. This symmetry is seen with many of the EPP reported points and is to be expected because the wind forecast error tested was also nearly symmetric. The convergence of the cross-track errors at zero time horizon is again a result of the FMS wind blending. Trajectory points whose errors do not converge are those in the descent phase of flight, where the trajectory re-computations are suppressed in this FMS simulation. The predicted cross-track error is related to the predicted fly-by radius, which changes as the predicted wind at a point changes, especially in the wind-blending region of the trajectory.

Figure 20 shows the EPP vertical errors for all route lengths and all route points of the three wind magnitude conditions tested. The larger differences in vertical error between these runs can be seen at the points that are in the descent profile and prior to the first hard altitude constraint (e.g., BURTT, xOVER). We note that these un-constrained points exhibit some vertical error variation due to wind blending as well as the trajectory update that occurs with the discontinuity of each point's error profile at approximately 1800 seconds time horizon. In general, the vertical errors are symmetric with respect to

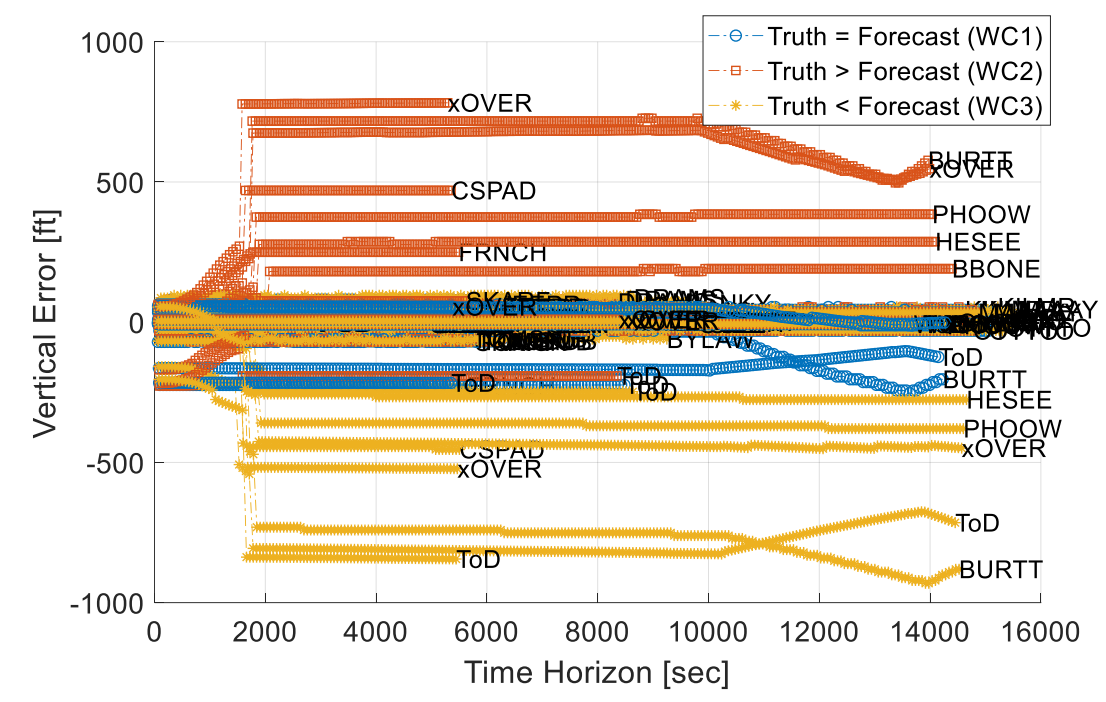

Figure 20. EPP vertical error for the perfect wind condition (WC1), the true wind magnitude greater than forecast (WC2), and the true wind magnitude less than forecast (WC3) [RL1-RL3, RT1, No RTA]. the perfect wind scenario and are larger outside the wind blending region.

Figure 21 shows the EPP time errors for all route lengths and all route points of the three wind magnitude conditions tested. As expected, the time errors are nearly symmetrical, with respect to the perfect wind condition, and decrease as the aircraft approaches each point. The time errors reach a non-zero constant value for waypoints in the descent phase of flight due to the lack of FMS trajectory updates in this region. This non-zero error is 15 seconds or less for the condition with truth wind magnitude greater than forecast (headwind stronger than forecast leads to late actual waypoint crossing times) and greater than -25 seconds for the condition with truth wind magnitude smaller than forecast (headwind weaker than forecast leads to early actual waypoint crossing times).

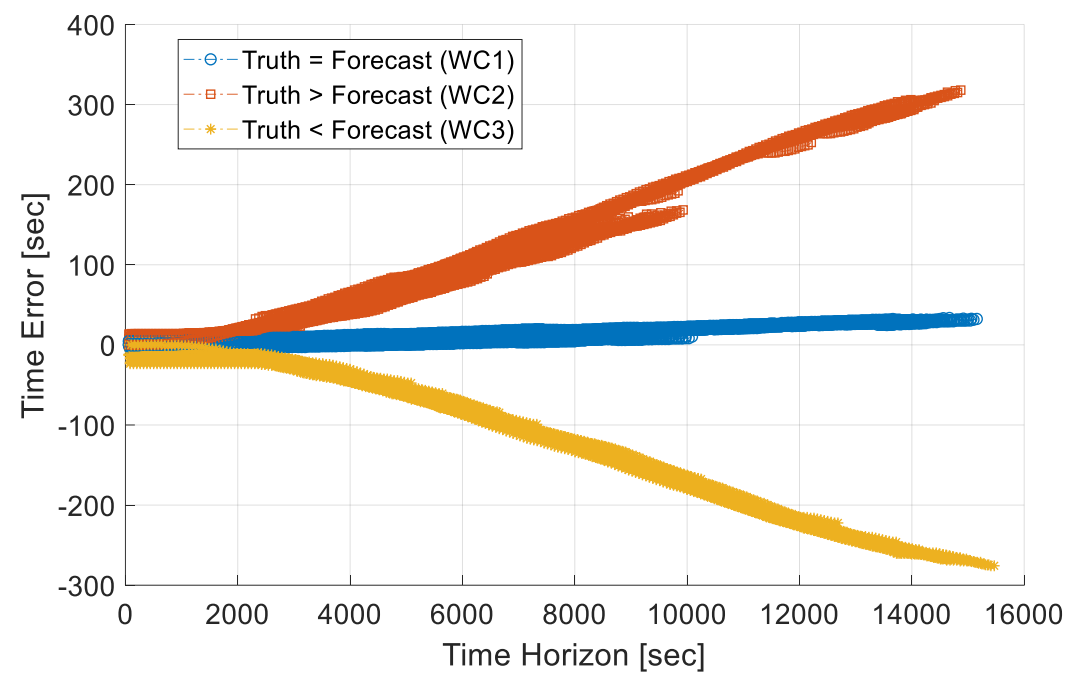

Figure 21. EPP time error for the perfect wind condition (WC1), the true wind magnitude greater than forecast (WC2), and the true wind magnitude less than forecast (WC3) [RL1-RL3, RT1, No RTA].

\section{E. Wind Direction Error Effects}

The EPP cross-track errors for the six wind-direction error scenarios (scenarios 1, and 6-10) tested can be seen in Figure 22. For the majority of the points, the cross-track errors are larger at large time horizons as the wind direction 
error increases. As the time horizon decreases, the cross-track errors converge to the cross-track error in the perfect wind condition. This convergence is the result of the FMS wind blending, which corrects for the wind forecast error as the flight approaches a waypoint. The cross-track error trends are caused by the prediction error in the groundspeeds

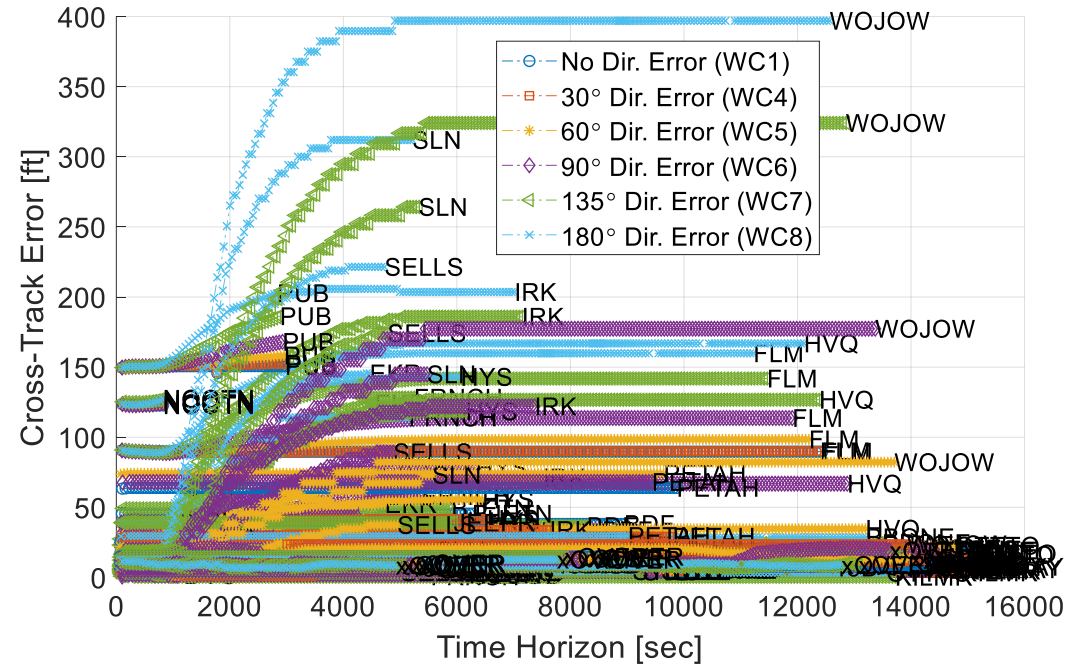

Figure 22. Cross-track error for EPP points of all route lengths with the perfect wind condition (WC1) and several wind direction error conditions (WC4-WC8) [RL1-RL3, RT1, No RTA].

at each waypoint, which, in turn, affect the predicted turn radius for those points. As an example, WOJOW has a predicted turn radius of 17.1 NM and a cross-track error of less than 10 feet in the perfect wind condition case. In the condition with 180 degrees of wind direction error, WC8, WOJOW has a reported turn radius of $22.4 \mathrm{NM}$ and cross-track error of 397 feet at long time horizons that converge to the radius and cross-track error of the perfect wind condition. The difference in radius for this 17.7degree turn at the long time horizon between these two conditions can be shown to contribute approximately 388 feet of additional cross-track error. The cross-track errors are also smaller for lower altitude points with speed constraints, where the groundspeed predictions have smaller magnitude errors.

The vertical errors for the six wind-direction forecast error scenarios can be seen in Figure 23. The trends in the vertical profile error are similar to those seen in the cross-track error, where the larger wind direction errors lead to the largest vertical errors. We do note that, for some points, such as BURTT and PHOOW, the vertical errors for the 135-degree direction error condition are slightly larger than the vertical errors for the 180-degree direction error condition. This is because that portion of the long route is better aligned with the 135-degree error wind direction thereby resulting in slightly higher forecast tailwind than in the 180-degree direction error condition.

The vertical errors are largest for the descent points just prior to the first "AT" altitude-constrained waypoint. The different winddirection error forecasts result in different forecast tailwind magnitude, which affect the groundspeed predictions and vertical path predictions at those unconstrained waypoints. The vertical errors in the constrained portion of the vertical path are small and nearly

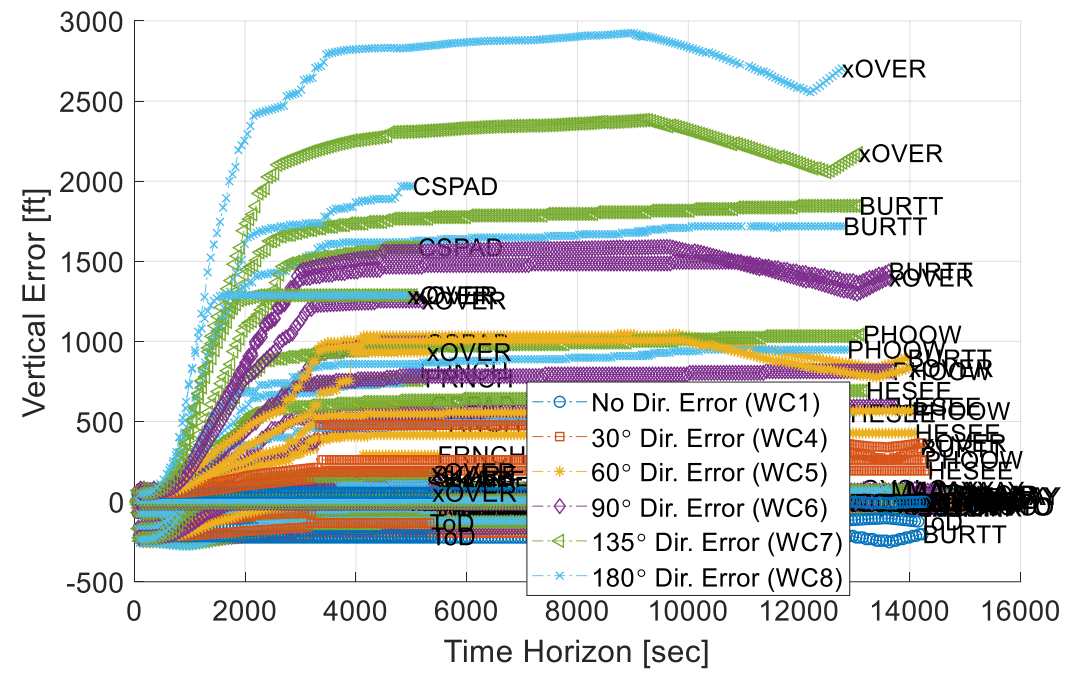

Figure 23. Vertical error for EPP points for all routes with the perfect wind condition (WC1) and several wind direction error conditions (WC4WC8) [RL1-RL3, RT1, No RTA]. identical in all wind direction error conditions.

In the wind conditions tested, the vertical error is generally positive at the long time horizons because the wind forecast simulates increasing tailwind as the wind direction error increases. The increasing tailwind has the effect of 
moving the predicted top-of-descent point earlier in the flight, thereby resulting in a predicted path that is below the actual path flown in the presence of the true winds.

The time errors for the six winddirection forecast error scenarios can be seen in Figure 24. As the wind direction forecast error increases from zero to 180 degrees, the error at long time horizons also increases. Similar to the vertical error trends, this is due to the increasing forecast tailwind that produces decreasingly earlier predicted crossing times at each waypoint as compared to the actual waypoint crossing times. As such, the time errors are generally positive, indicating later waypoint crossing times when compared with the predictions.

There is a nearly linear increase in time error as the time horizon increases. This is especially true for regions outside any wind blending horizon (time horizon greater than

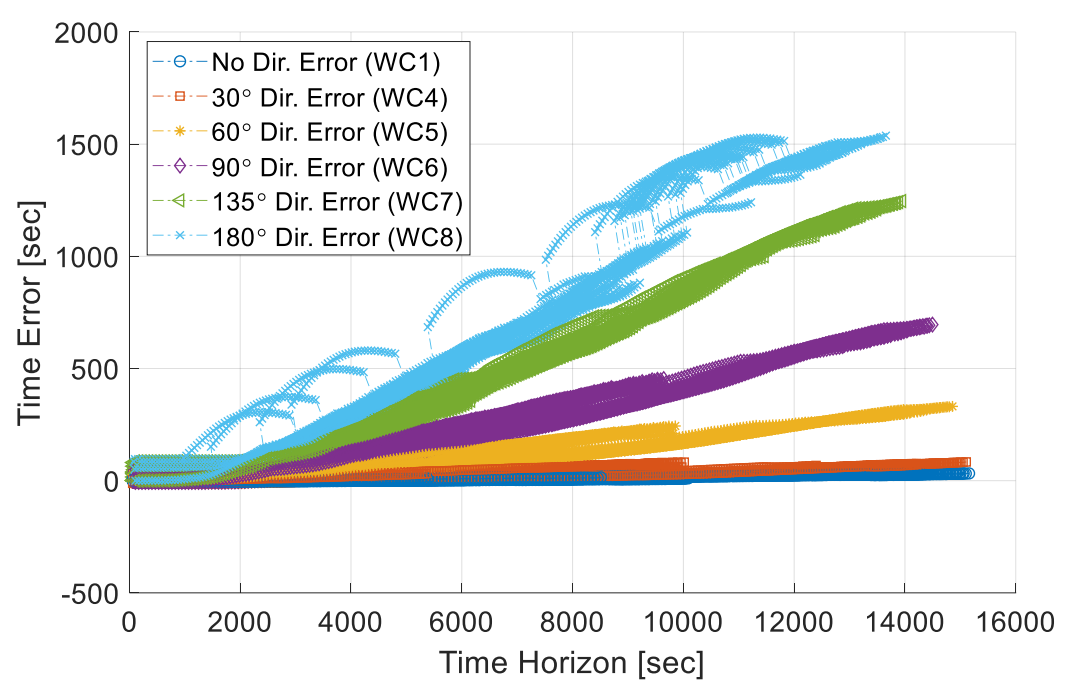

Figure 24. Time error for EPP points for all route lengths with the perfect wind condition (WC1) and several wind direction error conditions (WC4-WC8) [RL1-RL3, RT1, No RTA].

$\sim 5000$ seconds). The time errors approach zero as the time horizon approaches zero for en route waypoints. Descent waypoints reach a non-zero time error that is the time error at the last trajectory prediction just prior to the top-ofdescent point.

The "lobes" observed on the 180-degree wind direction forecast error are an artifact of the FMS's wind blending. They occur when the current track of the aircraft causes a wind direction interpolation that is to the right of course in on one leg and to the left of course on the next leg. This causes a discontinuity in the predicted wind magnitude that results in the discontinuity observed in the time errors and is a simulation artifact.

\section{F. Impact of Time Constrained Routes}

In this section, we investigate the impact of RTA waypoints on the EPP errors. Three different conditions were tested with each of the route lengths and compared with the equivalent non-RTA scenario: an RTA just prior to the top-of-descent point (RTA1, scenario 11), and RTA just after the top-of-descent point (RTA2, scenario 12), and an RTA close to the terminal area (RTA3, scenario 13). The baseline scenario for comparison is the scenario with a wind magnitude greater than the forecast magnitude (scenario 3).

The RTA capability of the RPFMS, and the assumptions of that capability, have an impact on the EPP errors. In the absence of an RTA, the FMS updates the reference trajectory at a frequency of once per minute. This update to the reference trajectory assumes the aircraft is following the FMS programmed speed profile. However, when an RTA exists on the active route, the reference trajectory is updated much less frequently, and each update reflects a change to the FMS speed profile necessary to correct any RTA error. The reference trajectory is only updated when a provisional trajectory prediction indicates that the estimated time at the RTA point is outside the allowable tolerance. This less frequent update to the reference trajectory in the presence of an RTA serves as a dead-band to prevent reactionary speed changes from occurring too frequently as the result of wind prediction and guidance errors, and to reduce the number of changes in the descent vertical path. In the ATOS simulation used here, the EPP message is composed from this infrequently updated reference trajectory, which leads to significant differences in the EPP error characteristics for a scenario with an RTA waypoint as compared to a scenario without an RTA waypoint.

Figure 25 shows the cross-track errors as a function of time horizon for the long route points and the baseline wind condition with no RTA (scenario 3) as well as the condition with an RTA just prior to the top-of-descent point (scenario 11). For scenario 11, the RTA waypoint is WOJOW. We can see that the condition with RTA has cross-track error profiles with long regions of constant cross-track error that are not seen in the baseline condition with no RTA. As 
explained above, this is related to the FMS's limited update rate for the reference trajectory, which leads to several EPP messages being generated from the same reference trajectory. We note that the magnitude of the cross-track error for the waypoint WOJOW at long time horizons is larger in the scenario with an RTA when compared to the no RTA scenario. In both scenarios, the cross-track error converges to less than 8 feet as the time horizon approaches zero. This larger error at long time horizons is related to a larger prediction error in the fly-by radius. The turn radius prediction at WOJOW in the RTA condition scenario increases from 17.3 to $19.5 \mathrm{NM}$ as the time horizon increases. Comparatively, in the non-RTA condition, the fly-by radius decreases from 17.9 to $17.1 \mathrm{NM}$ as the time horizon increases. In the non-RTA condition, the fly-by radius variation comes purely from groundspeed prediction error due to the wind forecast error in the presence of constant indicated cruise speed. In the RTA condition, however, the speed profile is allowed to vary to achieve the RTA and, combined with the wind forecast error, can lead to larger groundspeed variations that consequently produce larger fly-by radius variations. For example, in the non-RTA condition, the cruise speed is a constant Mach 0.801 , whereas, in the RTA condition, the initial Mach is 0.787 to meet the RTA but increases to Mach 0.843 just prior to crossing WOJOW to correct for the stronger than forecast headwind.

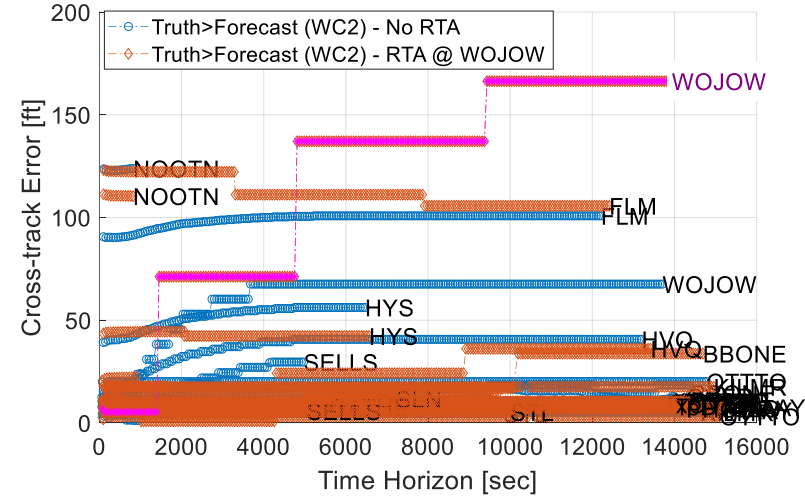

Figure 25. EPP cross-track error for the points of the long route with true wind magnitude greater than forecast condition, without RTA (No RTA) and with an RTA (RTA1) in the cruise portion of flight [RL1, RT1, WC2].

In Figure 26 we see the cross-track errors as a function of time horizon for the long route points and the baseline wind condition with no RTA (scenario 1) as well as the condition with an RTA just after to the top-of-descent point (scenario 12). In this RTA condition, the RTA waypoint is HESEE. In general, the RTA point, HESEE, does not have significant cross-track error as was seen in the point WOJOW. This is because HESEE has a small track angle change of less than one degree and no reported fly-by radius. We do note a large cross-track error at the xOVER point in the RTA condition at long time horizons because, in those trajectory predictions, the xOVER point falls on the arc of the turn at WOJOW and has cross-track error magnitudes close to those reported for WOJOW.

Figure 27 shows the cross-track errors as a function of time horizon for the long route points and the baseline wind condition with no RTA (scenario 1) as well as the condition with an RTA in the terminal area (scenario 13). In this RTA condition, the RTA waypoint is KILMR. KILMR also has very small, and nearly constant, cross-track error, which is the result of the speed and altitude constraint on that waypoint that limit the trajectory predictions of the FMS. 
Figure 28-Figure 30 show a comparison of the vertical errors between the baseline wind condition with no RTA (scenario 1) and the three RTA conditions (scenarios 11-13) for the points of the long route. All three RTA constrained waypoints show nearly zero vertical errors because WOJOW is a waypoint in the cruise phase of flight, where the altitude is nearly constant, and HESEE and KILMR both have altitude constraints on the descent profile. The waypoints with large vertical errors are those with no hard altitude constraints in the descent. There does not appear to be any clear distinction between the vertical errors when the RTA is in cruise versus when the RTA is in the descent portion of the flight.

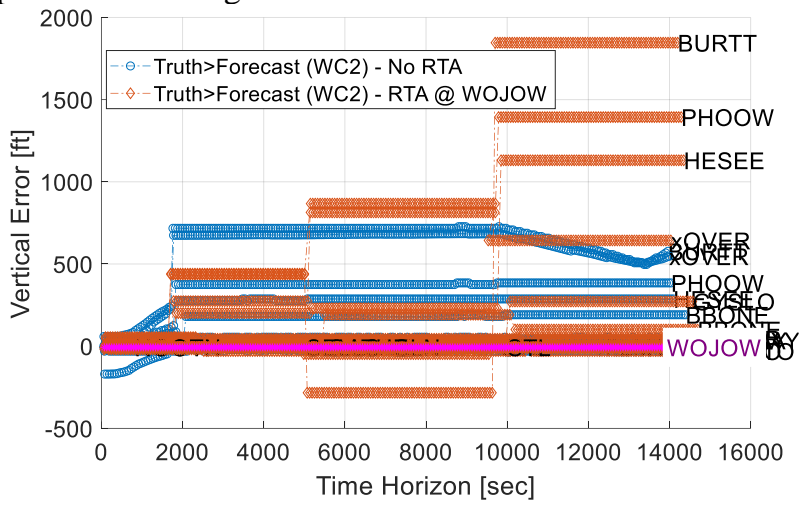

Figure 28. EPP vertical error for the points of the long route, with true wind magnitude greater than forecast condition, without RTA (No RTA) and with an RTA (RTA1) in the cruise portion of flight [RL1, RT1, WC2].

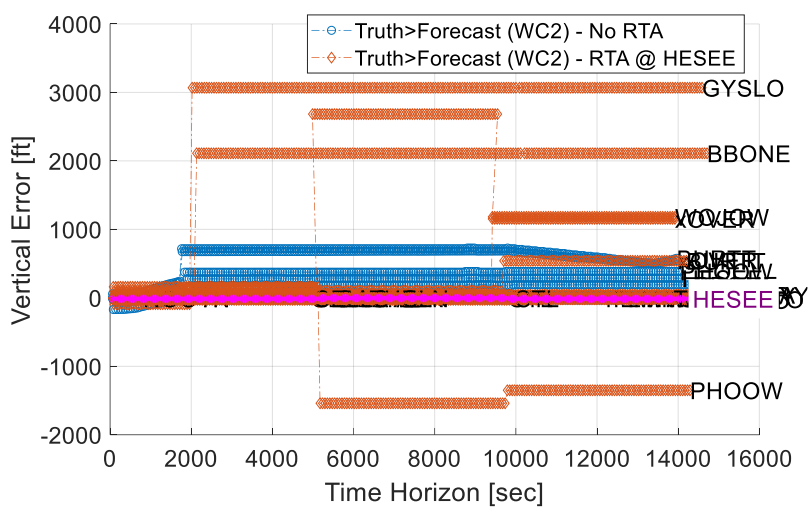

Figure 29. EPP vertical error for the points of the long route, with true wind magnitude greater than forecast condition, without RTA (No RTA) and with an RTA (RTA2) just after top-of-descent [RL1, RT1, WC2].

Figure 31-Figure 33 show a comparison of the time errors between the baseline wind condition with no RTA (scenario 1) and the three RTA conditions (scenarios 11-13) for the long route points. The time error for the RTA waypoints remains within the RTA tolerance of 30 seconds used in these scenarios. The time error for the remaining non-RTA waypoints, however, can be larger than in the condition when there is no RTA on the route. Even in regions of short time horizon, there are instances of waypoint crossing time error predictions that are larger than the non-RTA condition time errors for the same waypoint. Thus, perhaps intuitively, having an RTA on a route ensures small magnitude time errors for the RTA waypoint at the expense of larger time errors for the non-RTA waypoints. The time error profiles are nearly identical for the three RTA conditions.

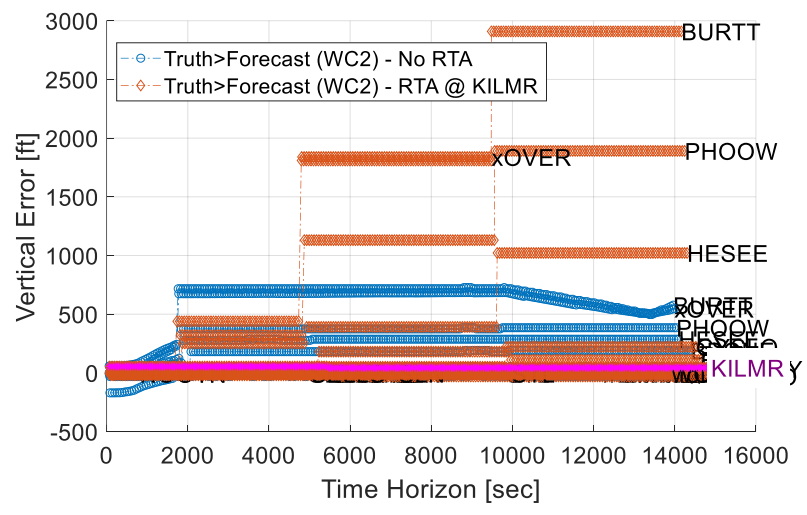

Figure 30. EPP vertical error for the points of the long route, with true wind magnitude greater than forecast condition, without RTA (No RTA) and with an RTA (RTA3) in the terminal area [RL1, RT1, WC2].

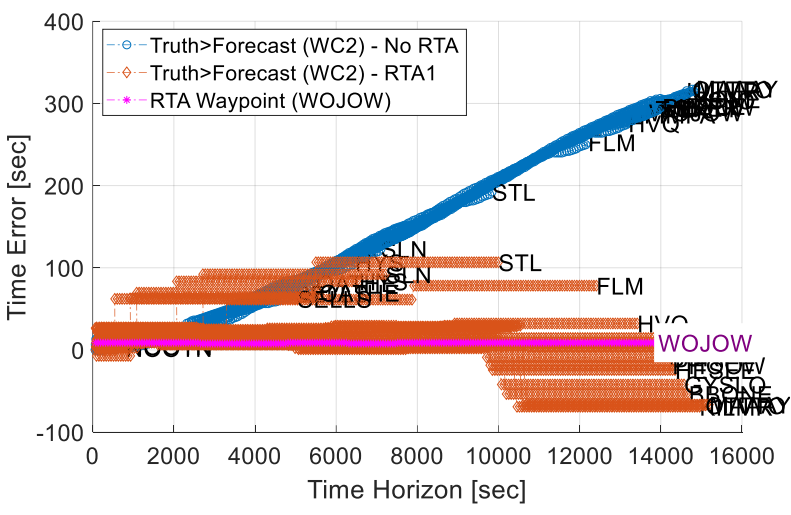

Figure 31. Time error for EPP points of the long route, true wind magnitude greater than forecast condition, without RTA (No RTA) and with an RTA (RTA1) in the cruise phase of flight [RL1, RT1, WC2]. 


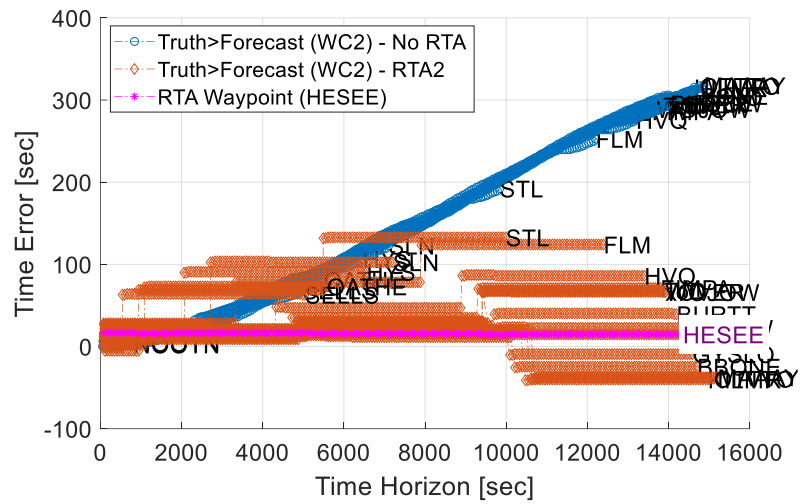

Figure 32. Time error for EPP points of the long route, true wind magnitude greater than forecast condition, without RTA (No RTA) and with an RTA (RTA2) in just after the top-of-descent [RL1, RT1, WC2].

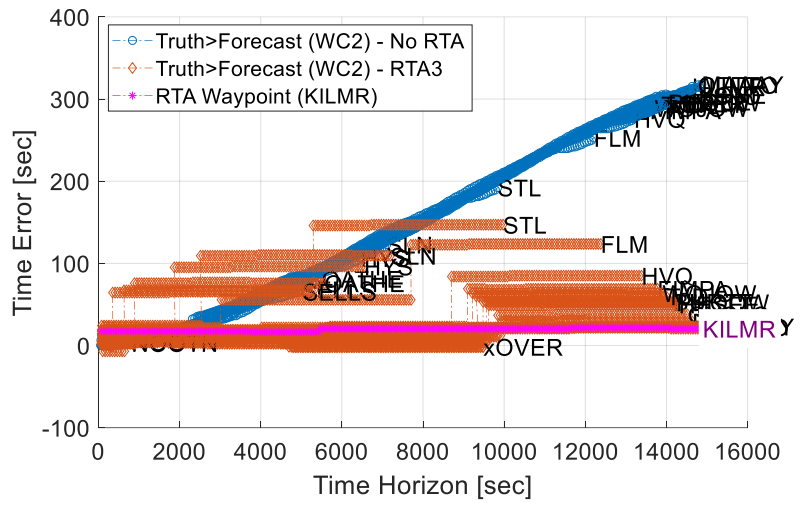

Figure 33. Time error for EPP points of the long route, true wind magnitude greater than forecast condition, without RTA (No RTA) and with an RTA (RTA3) close to the terminal area [RL1, RT1, WC2].

\section{Conclusion}

In this work, a set of scenarios were run in a medium-fidelity aircraft and FMS simulation to perform an initial characterization of EPP trajectory errors. Linear regression was also used to identify a set of EPP error models. Because this analysis was performed in a simulation environment, the results are highly dependent on the assumptions and algorithms within that environment and may not be directly applicable to the errors that may be present in the real system and with an operational FMS. In fact, there are many different types of FMS capabilities in the system today, each of which has different trajectory prediction capabilities and accuracies. A field investigation would be required to understand EPP errors under real conditions, thus qualifying this analysis as preliminary. Nonetheless, this analysis does point to some important characteristics of EPP trajectory errors.

The EPP errors were characterized under the following set of conditions: no wind, perfect wind, full versus sparse routes, with wind forecast magnitude error, with wind forecast direction error, and under RTA operations. The following is a set of observations that were gathered from the conditions simulated:

$>$ In the presence of no wind, when comparing EPP and FMS trajectory errors:

○ EPP cross-track errors can be as much as 100-200 feet larger than FMS errors, especially for large radius turns of en route waypoints, primarily due to MOT estimation

- EPP vertical errors differ from FMS errors only by the EPP altitude resolution of 10 feet

- EPP time errors differ from FMS errors only by the EPP time resolution of 1 second

$>$ In the absence of wind forecast error, EPP trajectory error magnitudes are:

- Less than 50 feet cross-track error for most points; on the order of 200 feet for large turn radius turns

- Less than 10 feet vertical error for most points; on the order of 200 feet for the top-of-descent point

- Within 30 seconds time error inside two hours of time horizon

In the presence of wind forecast error, EPP trajectory errors are:

- Related to the along-track wind forecast error

- Groundspeed affects the predicted turn radius

- Groundspeed affects the location of the ToD point and, thus, the descent vertical path

- Groundspeed affects the ETA at points

$\circ$ Reduced within the wind-blending region of the FMS

$>$ In the presence of RTA operations, EPP errors can be larger than without RTA, especially at long time horizons, due to the changing cost index and changing speed profile

$>$ Depending on the conditions, FMS wind blending can have a positive or a negative impact on the EPP trajectory errors 
$>$ EPP trajectory errors inherit the FMS's trajectory prediction errors but, in general, are larger than the FMS trajectory errors

$>$ EPP vertical errors are more pronounced for points in the descent profile prior to an altitude constrained point

This EPP error analysis was intended to be an initial characterization to produce an order of magnitude understanding and to identify further limitations in the use of the shared EPP trajectory information.

\section{References}

[1] NextGen Joint Planning and Development Office, "Concept of Operations for the Next Generation Air Transportation System, Version 3.2," JPDO, Washington, D.C., 2011.

[2] NextGen Joint Planning and Development Office, "JPDO Trajectory-Based Operations (TBO) Study Team Report," JPDO, Washington, D.C., 2011.

[3] RTCA, Inc., "DO-350A; Safety and Performance Requirements Standard for Baseline 2 ATS Data Communications," RTCA, Inc., Washington, 2016.

[4] Directorate-General for Energy and Transport, Data Link Services (DLS) Mandate, Brussel: European Commission, 2005.

[5] R. Šošovička, P. Veselý and J. Svoboda, "Estimation of Aircraft Performance Parameters from ADS-C EPP Data," in 2015 Integrated Communication, Navigation and Surveillance Conference (ICNS), Herndon, 2015.

[6] J. Bronsvoort, G. McDonald, M. Paglione, C. M. Young, A. Fabian, J. Boucquey and C. Garcia Avello, "Demonstration of Improved Trajectory Prediction Using Future Air Navigation Systems," Air Traffic Control Quarterly, vol. 21, no. 4, pp. 355-381, 2013.

[7] J. Bronsvoort, G. McDonald, J. Hochwarth and E. Gallo, "Air to Ground Trajectory Synchronisation through Extended Predicted Profile (EPP), A Pilot Study," in 14th AIAA Aviation Technology, Integration, and Operations Conference, Atlanta, 2014.

[8] E. Haugg, M. Poppe, S. Herr, T. Pütz, J. Svoboda and R. Šošovicka, "The Usability of ADS-C EPP Data for Air Traffic Control Applications," in 2015 IEEE/AIAA 34th Digital Avionics Systems Conference , Prague, 2015.

[9] J. Bronsvoort, G. McDonald, M. Paglione, C. M. Young, J. Boucquey, J. K. Hochwarth and E. Gallo, "Real-Time Trajectory Predictor Calibration through Extended Projected Profile Down-Link," in Eleventh USA/Europe Air Traffic Management Research and Development Seminar (ATM2015), Lisbon, 2015.

[10] J. Bronsvoort, G. McDonald, S. Torres, J. Hochwarth, J. Boucquey, M. Paglione, C. Young and M. Vilaplana, "Use of the Extended Projected Profile (EPP) in Trajectory Management," in 16th AIAA Aviation Technology, Integration, and Operations Conference, Washington, D.C., 2016.

[11] S. Mondoloni and I. Bayraktutar, "Impact Of Factors, Conditions, and Metrics On Trajectory Prediction Accuracy," in Sixth USA/Europe Air Traffic Management Research and Development Seminar (ATM2005), Baltimore, 2005.

[12] R. A. Vivona, K. T. Cate and S. M. Green, "Comparison of Aircraft Trajectory Predictor Capabilities and Impacts on Automation Interoperability," in 11th AIAA Aviation Technology, Integration, and Operations (ATIO) Conference, Virginia Beach, 2011.

[13] M. Paglione, I. Bayraktutar, G. McDonald and J. Bronsvoort, "Lateral Intent Error's Impact on Aircraft Prediction," Air Traffic Control Quarterly, vol. 18, no. 1, pp. 29-62, 2010.

[14] J. Bronsvoort, G. McDonald, M. Paglione, C. Garcia-Avello, I. Bayraktutar and C. M. Young, "Impact of Missing Longitudinal Aircraft Intent on Descent Trajectory Prediction," in 30th Digital Avionics Systems Conference, Seattle, 2011. 
[15] M. E. Peters, M. G. Ballin and J. S. Sakosky, "A Multi-Operator Simulation for Investigation of Distributed Air Traffic Management Concepts," in AIAA Modeling and Simulation Technologies conference and Exhibit, August 5-8, Monterey, California, 2002.

[16] N. M. Guerreiro and M. C. Underwood, "Preliminary Error Characterization and Parametric Error Model for the Automatic Dependent Surveillance - Contract Extended Projected Profile Message," NASA/TM2018-219810, Hampton, VA, 2018.

[17] S. Benjamin, C. Alexander, S. Weygandt, D. Dowell, M. Hu, T. Alcott, T. Smirnova, J. Olson, J. Brown, E. James and J. Kenyon, Improvements in HRRRv2/RAPv3 for Aug 2016 Implementation at NCEP for More Accurate Warm-Season and Cold-Season NWP, Salt Lake City: NOAA, 2016.

[18] RTCA, Inc., "DO-305A, Future Air Navigation System 1/A - Aeronautical Telecommunication Network Interoperability Standard (FANS 1/A - ATB B1 Interop Standard)," RTCA, Inc., Washinton, D.C., 2012.

[19] K. S. Linsday, "Results of a URET Operational Utility Experiment," MITRE Center for Advanced Aviation System Development, McLean, 2000.

[20] C. Querejeta, M. A. Vilaplana, E. Gallo, J. Lopez-Leones and F. A. Navarro, "Predicting Aircraft Trajectory". European Patent EP2040137B1, 16 May 2012.

[21] J. López-Leonés, M. A. Vilaplana, E. Gallo, F. A. Navarro and C. Querejeta, "The Aircraft Intent Description Language: A key enabler for Air-Ground synchronization in Trajectory-Based Operations," in 26th Digital Avionics Systems Conference, Dallas, 2007.

[22] J. Klooster, S. Torres, D. Earman, M. Castillo-Effen, R. Subbu, L. Kammer, D. Chan and T. Tomlinson, "Trajectory Synchronization and Negotiation in Trajectory Based Operations," in 29th Digital Avionics Systems Conference, Salt Lake City, 2010.

[23] M. T. Palmer and M. G. Ballin, "A High-Performance Simulated On-Board Avionics Architecture to Support Traffic Operations Research," in AIAA Modeling and Simulation Technologies Conference, Austin, 2003. 